\title{
Gluon propagator and three-gluon vertex with dynamical quarks
}

\author{
A. C. Aguilar ${ }^{1, a}$, F. De Soto ${ }^{2}$, M. N. Ferreira ${ }^{1}$, J. Papavassiliou ${ }^{3}$, J. Rodríguez-Quintero ${ }^{4}$, S. Zafeiropoulos ${ }^{5}$ \\ ${ }^{1}$ Institute of Physics “Gleb Wataghin”, University of Campinas-UNICAMP, 13083-859 Campinas, São Paulo, Brazil \\ 2 Dpto. Sistemas Físicos, Químicos y Naturales, Universidad Pablo de Olavide, 41013 Seville, Spain \\ ${ }^{3}$ Department of Theoretical Physics and IFIC, University of Valencia and CSIC, 46100 Valencia, Spain \\ ${ }^{4}$ Department of Integrated Sciences, University of Huelva, 21071 Huelva, Spain \\ ${ }^{5}$ Aix Marseille Univ, Université de Toulon, CNRS, CPT, Marseille, France
}

Received: 2 January 2020 / Accepted: 11 February 2020 / Published online: 20 February 2020

(C) The Author(s) 2020

\begin{abstract}
We present a detailed analysis of the kinetic and mass terms associated with the Landau gauge gluon propagator in the presence of dynamical quarks, and a comprehensive dynamical study of certain special kinematic limits of the three-gluon vertex. Our approach capitalizes on results from recent lattice simulations with $(2+1)$ domain wall fermions, a novel nonlinear treatment of the gluon mass equation, and the nonperturbative reconstruction of the longitudinal threegluon vertex from its fundamental Slavnov-Taylor identities. Particular emphasis is placed on the persistence of the suppression displayed by certain combinations of the vertex form factors at intermediate and low momenta, already known from numerous pure Yang-Mills studies. One of our central findings is that the inclusion of dynamical quarks moderates the intensity of this phenomenon only mildly, leaving the asymptotic low-momentum behavior unaltered, but displaces the characteristic "zero crossing" deeper into the infrared region. In addition, the effect of the three-gluon vertex is explored at the level of the effective gauge coupling, whose size is considerably reduced with respect to its counterpart obtained from the ghost-gluon vertex. The main upshot of the above considerations is the further confirmation of the tightly interwoven dynamics between the two- and three-point sectors of QCD.
\end{abstract}

\section{Introduction}

The three-gluon vertex of QCD [1-5], to be denoted by $\Gamma_{\alpha \mu \nu}$, has received particular attention in recent years because, in addition to its phenomenological relevance, it displays features that are inextricably connected with subtle dynamical mechanisms operating in the two-point sector of the theory. In particular, the emergence of a gluonic mass scale [6-12],

\footnotetext{
a e-mail: aguilar@ifi.unicamp.br (corresponding author)
}

in conjunction with the nonperturbative masslessness of the ghost field [13-16], would appear to account for the "infrared (IR) suppression" of the basic form factors of $\Gamma_{\alpha \mu \nu}$, established in lattice simulations [17-20] as well as in numerous continuum approaches [21-31].

The IR saturation of the Landau gauge gluon propagator [15,16,32-49], $\Delta\left(q^{2}\right)$, has been extensively studied within the framework developed from the fusion of the pinchtechnique (PT) $[6,50-52]$ with the background-field method (BFM) [53], known as the "PT-BFM scheme" [38,54]. From the dynamical point of view, the saturation is explained by implementing the Schwinger mechanism at the level of the SDE that controls the momentum evolution of $\Delta\left(q^{2}\right)[48,55]$. In this context, it is natural to regard $\Delta\left(q^{2}\right)$ as the sum of two distinct components, the "kinetic term", $J\left(q^{2}\right)$, and the (momentum-dependent) mass term, $m^{2}\left(q^{2}\right)$, as shown in Eq. (3.1). This splitting enforces a special realization of the Slavnov-Taylor identity (STI) satisfied by the fully dressed $\Gamma_{\alpha \mu \nu}$ [55], which allows the reconstruction of its longitudinal part by means of a nonperturbative generalization [31] of the well-known Ball-Chiu (BC) construction [2]. Specifically, the 10 longitudinal form factors of $\Gamma_{\alpha \mu \nu}$, to be denoted by $X_{i}$, are fully determined by the $J\left(q^{2}\right)$, the ghost dressing function, $F\left(q^{2}\right)$, and three of the five form factors comprising the ghost-gluon kernel, $H_{\mu \nu}[2,3,56]$. However, out of all these ingredients, it is the $J\left(q^{2}\right)$ that is largely responsible for the main qualitative characteristics of the $X_{i}$ [31].

As has been explained in earlier works, the SDE governing the $J\left(q^{2}\right)$ is composed by two types of (dressed) loops, those containing gluons with a dynamically generated mass scale, and those with massless ghosts [23]. The former furnish contributions that, due to the presence of the mass, are regulated in the IR, while the latter give rise to "unprotected" logarithms, of the type $\ln \left(q^{2} / \mu^{2}\right)$, which diverge as $q^{2} \rightarrow 0$. The combined effect of these terms is rather striking: as the (Euclidean) momentum $q^{2}$ decreases, $J\left(q^{2}\right)$ departs gradu- 
ally from its tree-level value (unity), reverses its sign ("zero crossing"), and finally diverges logarithmically at the origin [23]. Quite interestingly, the same overall pattern is displayed by the special combinations of vertex form factors studied in the (quenched) $\mathrm{SU}(2)$ lattice simulations of $[17,18]$ and SU(3) [19,20,57], exposing the deep connection between the two- and three-point sectors of the theory, encoded in the fundamental STIs.

To date, the three-gluon vertex studies carried out within the PT-BFM framework have been limited to the pure YangMills theory [31]. In the present work, we take a closer look at the structure of this vertex in the presence of dynamical quarks, thus making contact with real-world QCD.

In particular, we present and analyze results for $\Delta\left(q^{2}\right)$ and $\Gamma_{\alpha \mu \nu}$ obtained from numerical simulations of lattice QCD, using ensembles of gauge fields with $N_{f}=2+1$ domain wall fermions [58-60], at the physical point, $m_{\pi}=139 \mathrm{MeV}$. These lattice results are complemented by a detailed analysis based on the gluon SDE and the STIs that connect the kinetic term of $\Delta\left(q^{2}\right)$ with the form factors of $\Gamma_{\alpha \mu \nu}$; for brevity, we will refer to our continuum treatment as "SDE-based". Within this latter approach, the "unquenched" $J\left(q^{2}\right)$ is determined following the procedure first introduced in [61], using as aid the aforementioned lattice results for $\Delta\left(q^{2}\right)$. Then, the $J\left(q^{2}\right)$ is employed as the main ingredient of the nonperturbative $\mathrm{BC}$ construction introduced in [31], which provides definite predictions for the two special combinations of vertex form factors, denoted by $\bar{\Gamma}_{1}^{\mathrm{sym}}\left(q^{2}\right)$ and $\bar{\Gamma}_{3}^{\mathrm{asym}}\left(q^{2}\right)$, considered in our lattice simulation.

The main findings of this work may be summarized as follows. (i) There is excellent agreement between the SDEbased calculation and the lattice data for $\bar{\Gamma}_{1}^{\mathrm{sym}}\left(q^{2}\right)$ and $\bar{\Gamma}_{3}^{\text {asym }}\left(q^{2}\right)$. (ii) Given that all quark loops are tamed in the IR by the constituent quark masses, the logarithmic divergence displayed by $J\left(q^{2}\right)$ is still controlled by the ghost-loop, which is essentially insensitive to unquenching effects [36]. (iii) The deep IR behavior of $\bar{\Gamma}_{1}^{\text {sym }}\left(q^{2}\right)$ and $\bar{\Gamma}_{3}^{\text {asym }}\left(q^{2}\right)$ is determined by the corresponding asymptotic form of $J\left(q^{2}\right)$, multiplied by the value of the ghost dressing function at the origin, namely $F(0)$. (iv) The positions of the zero crossings displayed by the unquenched $J\left(q^{2}\right), \bar{\Gamma}_{1}^{\mathrm{sym}}\left(q^{2}\right)$, and $\bar{\Gamma}_{3}^{\text {asym }}\left(q^{2}\right)$ move deeper into the IR region with respect to the quenched cases, in agreement with the results reported in [28]. (v) The suppression of $J\left(q^{2}\right)$, and, correspondingly, of $\bar{\Gamma}_{1}^{\text {sym }}\left(q^{2}\right)$ and $\bar{\Gamma}_{3}^{\text {asym }}\left(q^{2}\right)$, is about $25 \%$ milder than in the quenched case.

The article is organized as follows. In Sect. 2 we introduce the necessary concepts and notation, and define the quantities studied in the lattice simulation. In Sect. 3 we present the salient theoretical notions associated with the gluon kinetic term, $J\left(q^{2}\right)$, and outline the procedure that permits us its indirect determination when dynamical quarks are included. Next, in Sect. 4 the SDE-based predictions for $\bar{\Gamma}_{1}^{\text {sym }}\left(q^{2}\right)$ and $\bar{\Gamma}_{3}^{\text {asym }}\left(q^{2}\right)$ are derived, and subsequently compared with the lattice results. Moreover, the corresponding running couplings are constructed, and directly compared with the corresponding quantity obtained from the ghost-gluon vertex. Finally, in Sect. 5 we discuss the results and summarize our conclusions.

\section{The three-gluon vertex: general considerations}

In this section we first present the basic definitions and conventions related with the gluon propagator and the threegluon vertex. Then, we review the two main quantities (vertex projections) that have been evaluated in the lattice simulation reported here.

\subsection{Notation and basic properties}

Throughout this article we work in the Landau gauge, where the gluon propagator is completely transverse,

$\Delta_{\mu \nu}^{a b}(p)=\left\langle\widetilde{A}_{\mu}^{a}(p) \widetilde{A}_{\nu}^{b}(-p)\right\rangle=\Delta\left(p^{2}\right) \delta^{a b} P_{\mu \nu}(p) ;$

$\widetilde{A}_{\mu}^{a}$ are the SU(3) gauge fields in Fourier space, the average $\langle\cdot\rangle$ indicates functional integration over the gauge space, and $P_{\mu \nu}(p)=g_{\mu \nu}-p_{\mu} p_{\nu} / p^{2}$.

In addition, we introduce the ghost propagator, $D^{a b}\left(q^{2}\right)=$ $\delta^{a b} D\left(q^{2}\right)$, related to its dressing function, $F\left(q^{2}\right)$, by

$D\left(q^{2}\right)=\frac{i F\left(q^{2}\right)}{q^{2}}$.

Similarly, in the three-point sector of QCD, one defines the correlation function of three gauge fields, at momenta $q$, $r$, and $p$ (with $q+r+p=0$ ),

$$
\begin{aligned}
\mathcal{G}_{\alpha \mu \nu}^{a b c}(q, r, p) & =\left\langle\widetilde{A}_{\mu}^{a}(q) \widetilde{A}_{\nu}^{b}(r) \widetilde{A}_{\rho}^{c}(p)\right\rangle \\
& =f^{a b c} \mathcal{G}_{\alpha \mu \nu}(q, r, p),
\end{aligned}
$$

where the connected three-point function $\mathcal{G}_{\alpha \mu \nu}(q, r, p)$ is given by

$$
\begin{aligned}
\mathcal{G}_{\alpha \mu \nu}(q, r, p)= & g \Gamma_{\alpha^{\prime} \mu^{\prime} v^{\prime}}(q, r, p) P_{\alpha}^{\alpha^{\prime}}(q) \\
& \times P_{\mu}^{\mu^{\prime}}(r) P_{v}^{v^{\prime}}(p) \Delta\left(q^{2}\right) \Delta\left(r^{2}\right) \Delta\left(p^{2}\right),
\end{aligned}
$$

with $\Gamma_{\alpha \mu \nu}(q, r, p)$ denoting the conventional one-particle irreducible (1-PI) three-gluon vertex (see Fig. 1).

It is customary to introduce the transversally projected vertex, $\bar{\Gamma}_{\alpha \mu \nu}(q, r, p)$, defined as [17]

$\bar{\Gamma}_{\alpha \mu v}(q, r, p)=\Gamma_{\alpha^{\prime} \mu^{\prime} v^{\prime}}(q, r, p) P_{\alpha}^{\alpha^{\prime}}(q) P_{\mu}^{\mu^{\prime}}(r) P_{v}^{v^{\prime}}(p)$,

such that

$\mathcal{G}_{\alpha \mu \nu}(q, r, p)=g \bar{\Gamma}_{\alpha \mu \nu}(q, r, p) \Delta\left(q^{2}\right) \Delta\left(r^{2}\right) \Delta\left(p^{2}\right)$. 


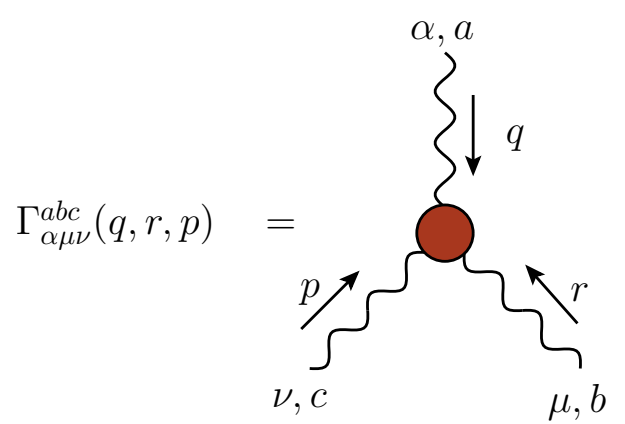

Fig. 1 The three-gluon vertex and the corresponding momentum/index conventions

Evidently,

$$
\begin{aligned}
q^{\alpha} \bar{\Gamma}_{\alpha \mu \nu}(q, r, p) & =r^{\mu} \bar{\Gamma}_{\alpha \mu \nu}(q, r, p) \\
& =p^{v} \bar{\Gamma}_{\alpha \mu \nu}(q, r, p)=0 .
\end{aligned}
$$

The vertex $\Gamma_{\alpha \mu \nu}(q, r, p)$ is usually decomposed into two distinct pieces, according to $[2,3,61]$,

$\Gamma^{\alpha \mu \nu}(q, r, p)=\Gamma_{L}^{\alpha \mu v}(q, r, p)+\Gamma_{T}^{\alpha \mu v}(q, r, p)$,

where the "longitudinal" part, $\Gamma_{L}^{\alpha \mu v}(q, r, p)$, saturates the corresponding STIs [see Eq. (3.5)], while the totally "transverse" part, $\Gamma_{T}^{\alpha \mu v}(q, r, p)$, satisfies Eq. (2.7).

The tensorial decomposition of $\Gamma_{L}^{\alpha \mu v}(q, r, p)$ and $\Gamma_{T}^{\alpha \mu \nu}(q, r, p)$ reads

$\Gamma_{L}^{\alpha \mu \nu}(q, r, p)=\sum_{i=1}^{10} X_{i}(q, r, p) \ell_{i}^{\alpha \mu \nu}$,

$\Gamma_{T}^{\alpha \mu \nu}(q, r, p)=\sum_{i=1}^{4} Y_{i}(q, r, p) t_{i}^{\alpha \mu \nu}$,

where the explicit expressions of the basis elements $\ell_{i}^{\alpha \mu v}$ and $t_{i}^{\alpha \mu \nu}$ are given in Eqs. (3.4) and (3.6) of [31], respectively.

It is clear that, due to Eq. (2.7), $\bar{\Gamma}_{\alpha \mu \nu}(q, r, p)$ may be expressed entirely in terms of the 4 tensors $t_{i}^{\alpha \mu \nu}$, i.e.,

$$
\begin{aligned}
& \bar{\Gamma}^{\alpha \mu v}(q, r, p) \\
& =\sum_{i=1}^{4}\left[Y_{i}(q, r, p)+\sum_{j=1}^{10} c_{i j} X_{j}(q, r, p)\right] t_{i}^{\alpha \mu \nu} .
\end{aligned}
$$

The presence of the $X_{j}(q, r, p)$ in the final answer may be understood by simply noticing that, after their transverse projection, the elements $\bar{\ell}_{i}^{\alpha \mu \nu}:=\ell_{i}^{\alpha^{\prime} \mu^{\prime} \nu^{\prime}} P_{\alpha^{\prime}}^{\alpha}(q) P_{\mu^{\prime}}^{\mu}(r) P_{v^{\prime}}^{\nu}(p)$, can be expressed as linear combinations of the $t_{i}^{\alpha \mu \nu}$; the exact expressions for the $c_{i j}$ may be straightforwardly worked out.

In addition, we define the tree-level analogue of Eq. (2.5),

$$
\bar{\Gamma}_{0}^{\alpha \mu \nu}(q, r, p)=\Gamma_{0}^{\alpha^{\prime} \mu^{\prime} v^{\prime}}(q, r, p) P_{\alpha^{\prime}}^{\alpha}(q) P_{\mu^{\prime}}^{\mu}(r) P_{v^{\prime}}^{\nu}(p),
$$

where

$$
\begin{aligned}
\Gamma_{0}^{\alpha \mu \nu}(q, r, p)= & (q-r)^{v} g^{\alpha \mu}+(r-p)^{\alpha} g^{\mu \nu} \\
& +(p-q)^{\mu} g^{\alpha \nu} .
\end{aligned}
$$

Note finally that, in the Euclidean space, the form factors $X_{i}(q, r, p)$ and $Y_{i}(q, r, p)$ are usually expressed as functions of $q^{2}, r^{2}$, and the angle $\theta$ formed between $q$ and $r$, namely $X_{i}(q, r, p) \rightarrow X_{i}\left(q^{2}, r^{2}, \theta\right)[31]$.

\subsection{The lattice observables}

The lattice two- and three-point correlation functions employed in the present work have been obtained from $N_{f}=2+1$ ensembles published in [58-60]; they were generated with the Iwasaki action for the gauge sector [62], and the Domain Wall action for the fermion sector $[63,64]$ (for related reviews, see, e.g., $[65,66])$. In order to reach the physical point, $m_{\pi}=139 \mathrm{MeV}$, the Möbius kernel [67] has been used, resulting in a simulation of light quarks with a mass ranging from 1.3 to $1.6 \mathrm{MeV}$, while the strange quark mass is $63 \mathrm{MeV}$; additional information on the particular setups is provided in Table 1 . Note that the data for the gluon propagator have been recently presented in [68], constituting a central ingredient in the construction of the process-independent QCD effective charge. In addition, in an earlier work [69], the same data were employed in the determination of the strong running coupling at the $Z^{0}$-boson mass within the so-called Taylor scheme. Finally, details on the Landau gauge computation of the gauge fields, and the correlation functions defined in Eqs. (2.1) and (2.3), may be found in [36,70]. In addition, the treatment of the $O(4)$-breaking artifacts has been carried out as described in [57,71-73].

Let us now consider the special quantity

$T(q, r, p)=\frac{W^{\alpha \mu \nu}(q, r, p) \mathcal{G}_{\alpha \mu \nu}(q, r, p)}{W^{\alpha \mu \nu}(q, r, p) W_{\alpha \mu \nu}(q, r, p)}$,

where the explicit form of the tensors $W_{\alpha \mu \nu}(q, r, p)$ will be judiciously chosen in order to project out particular components of the connected three-point function, $\mathcal{G}_{\alpha \mu \nu}(q, r, p)$, in certain simplified kinematic limits. Note that, in general, the quantity $T(q, r, p)$ is comprised of both longitudinal and transverse components, $X_{i}$ and $Y_{i}$.

As in [19], we focus on two special kinematic configurations:

(i) The totally symmetric limit, obtained when

$$
\begin{aligned}
& q^{2}=p^{2}=r^{2}:=s^{2}, \quad q \cdot p=q \cdot r=p \cdot r=-\frac{s^{2}}{2}, \\
& \theta=2 \pi / 3 .
\end{aligned}
$$


Table 1 Setup parameters for the four lattice ensembles used in this work

\begin{tabular}{llllll}
\hline$\beta$ & Size & $m_{\pi}(\mathrm{MeV})$ & $a^{-1}(\mathrm{GeV})$ & $\mathrm{V}\left(\mathrm{fm}^{4}\right)$ & Confs \\
\hline 2.37 & $32^{3} \times 64$ & 370 & 3.148 & $2.00^{3} \times 4.00$ & 590 \\
2.25 & $64^{3} \times 128$ & 139.15 & 2.359 & $5.35^{3} \times 10.70$ & 330 \\
2.13 & $48^{3} \times 96$ & 139.35 & 1.730 & $5.47^{3} \times 10.93$ & 350 \\
1.63 & $48^{3} \times 64$ & 137.5 & 0.997 & $9.43^{3} \times 12.57$ & 276 \\
\hline
\end{tabular}

(ii) The asymmetric limit, corresponding to the kinematic choice

$$
p \rightarrow 0, \quad r=-q, \quad \theta=\pi .
$$

Starting with case (i), it is relatively straightforward to establish that the application of the symmetric limit in Eq. (2.14) reduces the tensorial structure of $\bar{\Gamma}^{\alpha \mu \nu}(q, r, p)$ down to [19]

$$
\begin{aligned}
\bar{\Gamma}_{\mathrm{sym}}^{\alpha \mu \nu}(q, r, p)= & \bar{\Gamma}_{1}^{\mathrm{sym}}\left(s^{2}\right) \lambda_{1}^{\alpha \mu \nu}(q, r, p) \\
& +\bar{\Gamma}_{2}^{\mathrm{sym}}\left(s^{2}\right) \lambda_{2}^{\alpha \mu v}(q, r, p),
\end{aligned}
$$

with

$$
\begin{aligned}
& \lambda_{1}^{\alpha \mu v}(q, r, p)=\bar{\Gamma}_{0}^{\alpha \mu v}(q, r, p), \\
& \lambda_{2}^{\alpha \mu v}(q, r, p)=\frac{(r-p)^{\alpha}(p-q)^{\mu}(q-r)^{v}}{s^{2}} .
\end{aligned}
$$

The form factor $\bar{\Gamma}_{1}^{\mathrm{sym}}\left(s^{2}\right)$ is particularly interesting, because it captures certain exceptional features linked to a vast array of underlying theoretical ideas. $\bar{\Gamma}_{1}^{\text {sym }}\left(s^{2}\right)$ may be projected out by contracting Eq. (2.16) with the tensor

$\tilde{\lambda}_{1}^{\alpha \mu \nu}=\lambda_{1}^{\alpha \mu \nu}(q, r, p)+\frac{1}{2} \lambda_{2}^{\alpha \mu v}(q, r, p)$,

which is orthogonal to $\lambda_{2}^{\alpha \mu v}(q, r, p)$. Therefore, the substitution $W^{\alpha \mu \nu}(q, r, p) \rightarrow \tilde{\lambda}_{1}^{\alpha \mu v}(q, r, p)$ at the level of Eq. (2.13), and the subsequent implementation of Eq. (2.14) in the resulting expressions, leads to

$T^{\mathrm{sym}}\left(s^{2}\right):=\left.T(q, r, p)\right|_{\mathrm{Eq} .(2.14)} ^{W \rightarrow \widetilde{\lambda}_{1}}=g \bar{\Gamma}_{1}^{\mathrm{sym}}\left(s^{2}\right) \Delta^{3}\left(s^{2}\right)$.

As has been shown in [31], the use of the basis of Eq. (2.9) allows one to express $\bar{\Gamma}_{1}^{\text {sym }}\left(s^{2}\right)$ in the form

$\bar{\Gamma}_{1}^{\mathrm{sym}}\left(s^{2}\right)=X_{1}\left(s^{2}\right)-\frac{s^{2}}{2} X_{3}\left(s^{2}\right)+\frac{s^{4}}{4} Y_{1}\left(s^{2}\right)-\frac{s^{2}}{2} Y_{4}\left(s^{2}\right)$.

Turning to case (ii), the implementation of the asymmetric limit gives rise to an expression for $\bar{\Gamma}^{\alpha \mu \nu}(q, r, p)$ given by a single tensor, namely [19]

$\bar{\Gamma}_{\text {asym }}^{\alpha \mu \nu}(q, r, p)=\bar{\Gamma}_{3}^{\text {asym }}\left(q^{2}\right) \lambda_{1}^{\alpha \mu \nu}(q,-q, 0)$, with

$\lambda_{1}^{\alpha \mu \nu}(q,-q, 0)=2 q^{\nu} P^{\alpha \mu}(q)$.

Setting $W \rightarrow \lambda_{1}^{\alpha \mu v}(q,-q, 0)$ into Eq. (2.13), one obtains

$$
\begin{aligned}
T^{\operatorname{asym}}\left(q^{2}\right) & :=\left.T(q, r, p)\right|_{\text {Eq. }(2.15)} ^{W \rightarrow \lambda_{1}} \\
& =g \bar{\Gamma}_{3}^{\operatorname{asym}}\left(q^{2}\right) \Delta(0) \Delta^{2}\left(q^{2}\right) .
\end{aligned}
$$

Again, using Eq. (2.9), we may cast $\bar{\Gamma}_{3}^{\text {asym }}\left(q^{2}\right)$ in the form [31]

$\bar{\Gamma}_{3}^{\operatorname{asym}}\left(q^{2}\right)=X_{1}\left(q^{2}, q^{2}, \pi\right)-q^{2} X_{3}\left(q^{2}, q^{2}, \pi\right)$.

Interestingly, $\bar{\Gamma}_{3}^{\operatorname{asym}}\left(q^{2}\right)$ does not contain any reference to the transverse form factors $Y_{i}$, and may be therefore determined in its entirety by the nonperturbative $\mathrm{BC}$ construction of [31].

\section{The kinetic term of the gluon propagator}

In this section we take a closer look at the kinetic term of the gluon propagator, which, by virtue of the fundamental STIs, is closely connected with the longitudinal form factors $X_{i}$, introduced in Eq. (2.9). After reviewing certain salient theoretical concepts related to this quantity, we outline its indirect derivation from the unquenched gluon propagator and the corresponding gluon mass equation, and discuss some of its most outstanding properties.

\subsection{Basic concepts and key relations}

A special feature of $\Delta\left(q^{2}\right)$, observed in the Landau gauge, is its saturation in the deep IR $[6,15,74]$. This property has been firmly established in a variety of SU(2) $[32,75,76]$ and SU(3) [33-36,77-79] large-volume lattice simulations, both quenched and unquenched. Due to its far reaching theoretical implication, this property has been scrutinized in the continuum using a multitude of distinct approaches $[15,16,37$ 49,80-82].

This characteristic behavior of $\Delta\left(q^{2}\right)$ is considered to be intimately connected with the emergence of a gluon mass scale $[6,11,12]$, and has been studied in detail within the framework of the "PT-BFM" [38,54]. For the purposes of the present work, we will briefly comment on a limited number of concepts and ingredients related with this particular 
approach; for further details, the reader is referred to the extended literature cited above.

(a) The IR finiteness of $\Delta\left(q^{2}\right)$ motivates the splitting of its inverse into two separate components, according to (Euclidean space) [55]

$$
\Delta^{-1}\left(q^{2}\right)=q^{2} J\left(q^{2}\right)+m^{2}\left(q^{2}\right),
$$

where $J\left(q^{2}\right)$ corresponds to the so-called "kinetic term" [at tree-level, $J\left(q^{2}\right)=1$ ], while $m^{2}\left(q^{2}\right)$ to a momentumdependent gluon mass scale, with the property $m^{2}(0)=$ $\Delta^{-1}(0)$. Note that we have suppressed the dependence of all quantities appearing in Eq. (3.1) on the renormalization point $\mu$. For large values of $q^{2}$, the component $J\left(q^{2}\right)$ captures the standard perturbative corrections to the gluon propagator, while in the IR it exhibits exceptional nonperturbative features $[23,31]$.

(b) The emergence of the component $m^{2}\left(q^{2}\right)$ is triggered by the non-Abelian realization of the well-known Schwinger mechanism $[83,84]$ for gauge boson mass generation. This latter mechanism is activated through the inclusion of longitudinally coupled massless poles into the three-gluon vertex that enters in the SDE governing the evolution of $\Delta^{-1}\left(q^{2}\right)$ [55,85-88]. In particular, one implements the replacement

$\Gamma_{\alpha \mu \nu} \rightarrow \Gamma_{\alpha \mu \nu}=\Gamma_{\alpha \mu \nu}+V_{\alpha \mu \nu}$,

where $V_{\alpha \mu \nu}$ contains the aforementioned poles, arranged in the special tensorial structure [86]

$$
\begin{aligned}
V_{\alpha \mu \nu}(q, r, p)= & \left(\frac{q_{\alpha}}{q^{2}}\right) A_{\mu \nu}(q, r, p)+\left(\frac{r_{\mu}}{r^{2}}\right) B_{\alpha \nu}(q, r, p) \\
& +\left(\frac{p_{v}}{p^{2}}\right) C_{\alpha \mu}(q, r, p) .
\end{aligned}
$$

Consequently, by virtue of the relation $V_{\alpha^{\prime} \mu^{\prime} v^{\prime}}(q, r, p) P_{\alpha}^{\alpha^{\prime}}$ $(q) P_{\mu}^{\mu^{\prime}}(r) P_{v}^{v^{\prime}}(p)=0$, the component $V_{\alpha \mu \nu}(q, r, p)$ drops out from the quantity $T(q, r, p)$ defined in Eq. (2.13), and only the "no-pole" part of the vertex, $\Gamma_{\alpha \mu \nu}$, contributes to it.

(c) It turns out that the two functions composing $\Delta^{-1}\left(q^{2}\right)$ in Eq. (3.1) and the two vertices comprising $\Gamma_{\alpha \mu \nu}$ in Eq. (3.2) are firmly linked. Specifically, the STI satisfied by $\Gamma_{\alpha \mu \nu}(q, r, p)$,

$$
\begin{aligned}
q^{\alpha} \Gamma_{\alpha \mu \nu}(q, r, p)= & F\left(q^{2}\right)\left[\Delta^{-1}\left(p^{2}\right) P_{\nu}^{\alpha}(p) H_{\alpha \mu}(p, q, r)\right. \\
& \left.-\Delta^{-1}\left(r^{2}\right) P_{\mu}^{\alpha}(r) H_{\alpha \nu}(r, q, p)\right],
\end{aligned}
$$

is naturally separated into two "partial" ones, relating the divergences of $\Gamma_{\alpha \mu \nu}$ and $V_{\alpha \mu \nu}$ with $J\left(q^{2}\right)$ and $m^{2}\left(q^{2}\right)$, respectively, namely ${ }^{1}$

$$
\begin{aligned}
q^{\alpha} \Gamma_{\alpha \mu \nu}(q, r, p)= & F\left(q^{2}\right)\left[p^{2} J\left(p^{2}\right) P_{\nu}^{\alpha}(p) H_{\alpha \mu}(p, q, r)\right. \\
& \left.-r^{2} J\left(r^{2}\right) P_{\mu}^{\alpha}(r) H_{\alpha \nu}(r, q, p)\right],
\end{aligned}
$$

\footnotetext{
${ }^{1}$ Exactly analogous relations hold for the STIs with respect to the other two legs.
}

$$
\begin{aligned}
q^{\alpha} V_{\alpha \mu \nu}(q, r, p)= & F\left(q^{2}\right)\left[m^{2}\left(r^{2}\right) P_{\mu}^{\alpha}(r) H_{\alpha \nu}(r, q, p)\right. \\
& \left.-m^{2}\left(p^{2}\right) P_{\nu}^{\alpha}(p) H_{\alpha \mu}(p, q, r)\right] .
\end{aligned}
$$

The practical implication of this separation is that the form factors $X_{i}$ of $\Gamma_{L}^{\alpha \mu \nu}(q, r, p)$ may be reconstructed by means of a nonperturbative generalization [31] of the well-known BC procedure [2]. In particular, the $X_{i}$ are expressed as combinations of the $J\left(q^{2}\right)$, the ghost dressing function, $F\left(q^{2}\right)$, and three of the five components appearing in the tensorial decomposition of $H_{\mu \nu}$, whose one-loop dressed approximation has been computed in [56]. These results are especially relevant for the study in hand, because they provide a theoretical (albeit approximate) handle on the form of the $X_{i}$ appearing in Eqs. (2.20) and (2.24); note, however, that the $Y_{i}$ remain undetermined by this procedure.

(d) The special realization of the STIs explained in point (c) leads to the separation of the original SDE governing $\Delta\left(q^{2}\right)$ into a system of two coupled integral equations, one determining $J\left(q^{2}\right)$ and the other $m^{2}\left(q^{2}\right)$ [55]. As has been demonstrated recently in [61], the self-consistent treatment of the equation controlling $m^{2}\left(q^{2}\right)$, in conjunction with the (quenched) lattice data for $\Delta\left(q^{2}\right)$, permits one to pin down the form of $J\left(q^{2}\right)$ quite accurately, without actually invoking its own (considerably more complicated) integral equation. The subsequent use of this $J\left(q^{2}\right)$ as ingredient in the BC construction of the $X_{i}$ described above, allows one to obtain, through Eqs. (2.20) and (2.24), SDE-derived predictions for $T^{\mathrm{sym}}\left(s^{2}\right)$ and $T^{\text {asym }}\left(q^{2}\right)$, which are in excellent agreement with the lattice data of [19].

\subsection{The "unquenched" $J\left(q^{2}\right)$ : general construction and} main results

The above considerations, and in particular the procedure summarized in point (d), will be applied in the present work in order to obtain SDE-derived predictions for the unquenched $\bar{\Gamma}_{1}^{\text {sym }}\left(s^{2}\right)$ and $\bar{\Gamma}_{3}^{\text {asym }}\left(q^{2}\right)$, which will be subsequently compared with the corresponding sets of lattice results. In what follows we outline the main points of this construction, postponing the multitude of technical details for a future communication.

$\left(\mathrm{P}_{1}\right)$ : The starting point is the gluon mass equation considered in [61], whose general form is given by $\left(\alpha_{s}:=g^{2} / 4 \pi\right)$

$m^{2}\left(q^{2}\right)=\int \frac{\mathrm{d}^{4} k}{(2 \pi)^{4}} m^{2}(k) \Delta(k) \Delta(k+q) \mathcal{K}\left(k, q, \alpha_{s}\right)$,

where the kernel $\mathcal{K}$ receives one-loop and two-loop dressed contributions.

$\left(\mathrm{P}_{2}\right)$ : The effective treatment of multiplicative renormalization amounts to the substitution of the vertex renormalization constants, multiplying the one- and two-loop components of $\mathcal{K}$, by kinematically simplified form factors of the 
three- and four-gluon vertices, denoted by $\mathcal{C}_{3}\left(k^{2}\right)$ and $\mathcal{C}_{4}\left(k^{2}\right)$, respectively.

$\left(\mathrm{P}_{3}\right)$ : The kinetic term $J\left(q^{2}\right)$ enters into the gluon mass equation when the substitution given in Eq. (3.1) is implemented at the level of the term $\Delta(k) \Delta(k+q)$. In addition, the function $\mathcal{C}_{3}\left(k^{2}\right)$ depends on $J\left(k^{2}\right)$; specifically, for its derivation we adopt the Abelian version of the $\mathrm{BC}$ construction [2], setting the ghost dressing function and the ghostgluon kernel at their tree-level values, which yields simply $\mathcal{C}_{3}\left(k^{2}\right)=J\left(k^{2}\right)$.

$\left(\mathrm{P}_{4}\right)$ : The term $\mathcal{C}_{4}\left(k^{2}\right)$ is approximated by the same functional form given in Eq. (4.8) of [61]. As explained there, the main feature of $\mathcal{C}_{4}\left(k^{2}\right)$, which is instrumental for the stability of the gluon mass equation, is its mild enhancement with respect to its tree-level value in the critical region of a few hundred $\mathrm{MeV}$.

$\left(\mathrm{P}_{5}\right)$ : An initial Ansatz for $J\left(q^{2}\right)$ is introduced as a "seed", and is subsequently improved by means of a well-defined iterative procedure, described in detail in Sec. VB of [61]. In particular, both the form of $J\left(q^{2}\right)$ and the value of $\alpha_{s}$ are gradually modified, and each time the corresponding solution, $m^{2}\left(q^{2}\right)$, obtained from the gluon mass equations, is recorded. The procedure terminates when the pair $\left\{m^{2}\left(q^{2}\right), J\left(q^{2}\right)\right\}$ has been identified which, when combined according to Eq. (3.1), provides the best possible coincidence with the lattice data for $\Delta\left(q^{2}\right)$ with $N_{f}=2+1$ (see the left bottom panel of Fig. 2). The final value of the gauge charge is $\alpha_{s}=0.27$.

An excellent fit for $m^{2}\left(q^{2}\right)$, shown in the top left panel of Fig. 2, is given by

$m^{2}\left(q^{2}\right)=\frac{m_{0}^{4}}{\kappa_{1}^{2}+q^{2} \ln \left[\left(q^{2}+\kappa_{2}^{2}\right) / \sigma^{2}\right]}$,

where the parameters are given by $m_{0}^{4}=0.134 \mathrm{GeV}^{4}, \kappa_{1}^{2}=$ $0.705 \mathrm{GeV}^{2}, \kappa_{2}^{2}=9.31 \mathrm{GeV}^{2}$, and $\sigma^{2}=5.13 \mathrm{GeV}^{2}$.

Similarly, the solution for $J\left(q^{2}\right)$, shown in the top right panel of Fig. 2, is accurately fitted by

$$
\begin{aligned}
J\left(q^{2}\right)= & +\frac{3 \lambda_{s}}{4 \pi}\left(1+\frac{\tau_{1}}{q^{2}+\tau_{2}}\right)\left[2 \ln \left(\frac{q^{2}+\eta^{2}\left(q^{2}\right)}{\mu^{2}+\eta^{2}\left(\mu^{2}\right)}\right)\right. \\
& \left.+\frac{1}{6} \ln \left(\frac{q^{2}}{\mu^{2}}\right)\right],
\end{aligned}
$$

with

$\eta^{2}\left(q^{2}\right)=\frac{\eta_{1}}{q^{2}+\eta_{2}}$,

where $\lambda_{s}=0.237, \tau_{1}=7.06 \mathrm{GeV}^{2}, \tau_{2}=0.709 \mathrm{GeV}^{2}$, $\eta_{1}=22.35 \mathrm{GeV}^{4}, \eta_{2}=1.19 \mathrm{GeV}^{2}$, and $\mu^{2}=18.64$ $\mathrm{GeV}^{2}$. Notice that $J\left(\mu^{2}\right)=1$, as required by the momentum subtraction (MOM) renormalization prescription.

We emphasize that, even though several aspects of the unquenched gluon propagator have been previously addressed within the PT-BFM formalism ${ }^{2}$ [92,93], the results presented in Eqs. (3.8) and (3.9) are completely new.

\subsection{Asymptotic analysis for the deep IR}

By expanding the above fits for $J\left(q^{2}\right)$ and $m^{2}\left(q^{2}\right)$ around $q^{2} \rightarrow 0$, we obtain

$J\left(q^{2}\right)=a \ln \left(\frac{q^{2}}{\mu^{2}}\right)+b, \quad m^{2}\left(q^{2}\right)=d+c q^{2}$,

and therefore

$\Delta^{-1}\left(q^{2}\right)=d+q^{2}\left[a \ln \left(\frac{q^{2}}{\mu^{2}}\right)+b+c\right]$,

with

$$
\begin{aligned}
& a=\frac{\lambda_{s}}{8 \pi}\left(1+\frac{\tau_{1}}{\tau_{2}}\right), \\
& b=1+\frac{3 \lambda_{s}}{2 \pi}\left(1+\frac{\tau_{1}}{\tau_{2}}\right) \ln \left[\frac{\eta_{1}}{\eta_{2}\left[\mu^{2}+\eta^{2}\left(\mu^{2}\right)\right]}\right], \\
& c=-\frac{m_{0}^{4}}{\kappa_{1}^{4}} \ln \left(\frac{\kappa_{2}^{2}}{\sigma^{2}}\right), \quad d=\frac{m_{0}^{4}}{\kappa_{1}^{2}} .
\end{aligned}
$$

Employing the numerical values of the parameters in Eqs. (3.9) and (3.10), one obtains $a=0.104, b=0.934$, $c=-0.160, d=0.190 \mathrm{GeV}^{2}$.

With the above asymptotic expressions at our disposal, we proceed to elaborate on the following important points.

(i) As can be seen in the bottom right panel of Fig. 2, for momenta lower than about $500 \mathrm{MeV}$, the quenched and unquenched $J\left(q^{2}\right)$ run nearly parallel to each other. In view of Eq. (3.11), this indicates that the coefficient of the logarithm remains practically unchanged in the presence of quark loops, whose net effect in the deep IR is to simply modify (increase) the numerical value of the constant $b$, thus shifting the position of the zero crossing towards lower momenta. A qualitative explanation of these observations may be given by noting that (a) the ghost dressing function is rather insensitive to unquenching effects [36], and hence, the contribution of the ghost loops is essentially the same, and (b) the quark loops provide IR finite contributions, since the corresponding logarithms are protected by the quark masses; their size and sign is consistent with the analysis presented in [92]. It is important to emphasize, however, that throughout our present derivation, no quark loops have been actually evaluated; instead, by means of the optimization procedure described in $\left(\mathrm{P}_{5}\right)$, the effects of the dynamical quarks, implicit in the lattice data for $\Delta\left(q^{2}\right)$, have been indirectly transmitted to the individual components $J\left(q^{2}\right)$ and $m^{2}\left(q^{2}\right)$.

(ii) From Eq. (3.11) we can obtain a particularly accurate estimate of the position of the "zero crossing", i.e., the

\footnotetext{
${ }^{2}$ For related works, see also, e.g., [28,89-91].
} 

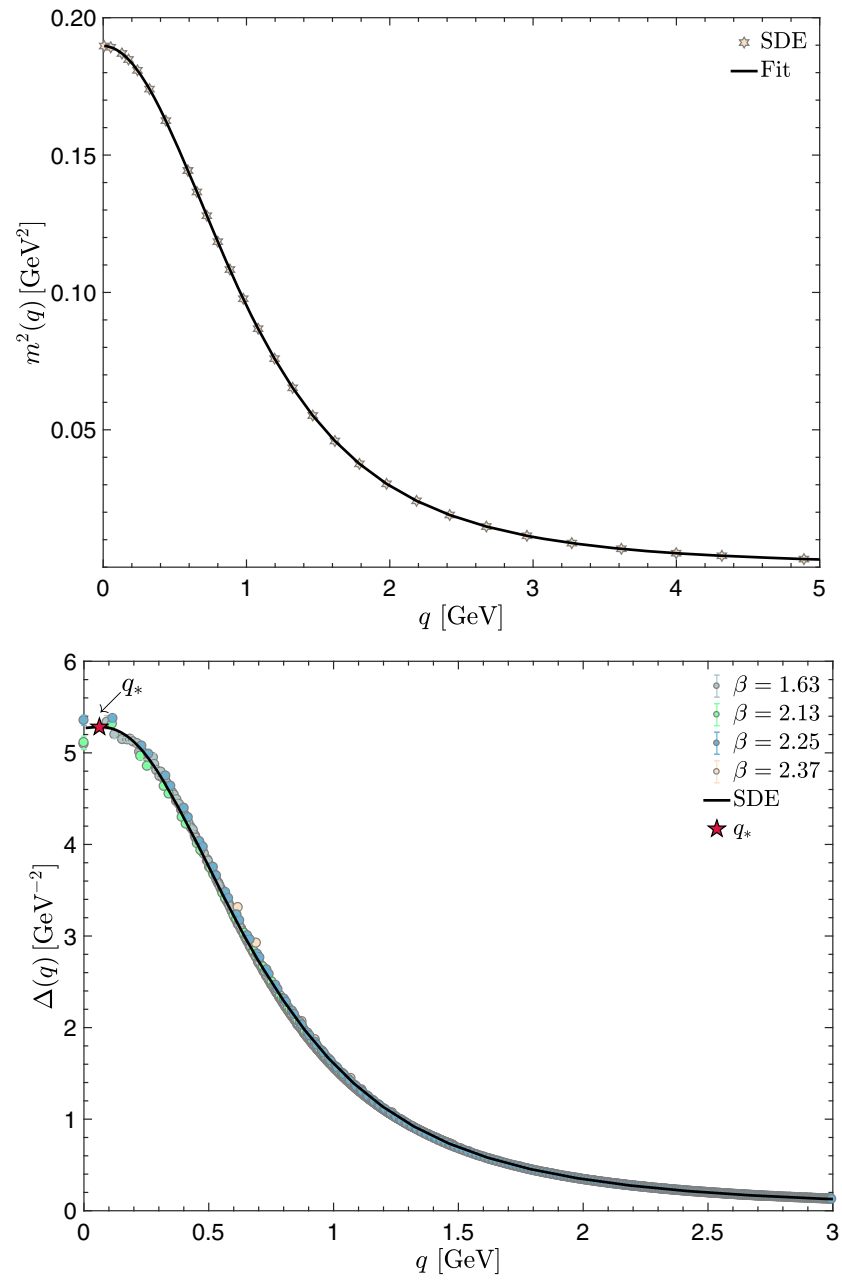

Fig. 2 Top left panel: the dynamical gluon mass, $m^{2}(q)$, obtained from Eq. (3.7) and fitted by Eq. (3.8). Top right panel: the $J(q)$ obtained through the procedure described in points $\left(P_{1}\right)-\left(P_{5}\right)$, and the corresponding fit, given in Eq. (3.9). Bottom left panel: Comparison of the unquenched gluon propagator, $\Delta(q)$, obtained from Eq. (3.1)

momentum $q_{0}$ for which $J\left(q_{0}\right)=0$; it is given by

$q_{0}=\mu e^{-\frac{b}{2 a}}$.

With the values of the coefficients found before, this leads to $q_{0}=48.19 \mathrm{MeV}$. On the other hand, computing the crossing of the full fit of Eq. (3.9) numerically yields $q_{0}=47.18 \mathrm{MeV}$ (see the red star in the bottom right panel of Fig. 2). Thus, the asymptotic form is accurate to within $0.3 \%$ for the position of the crossing of $J\left(q^{2}\right)$.

(iii) Let us next consider the maximum of $\Delta\left(q^{2}\right)$, and denote by $q_{*}$ the momentum where it occurs, namely the solution of the condition $\Delta^{\prime}\left(q^{2}\right)=0$, where the "prime" denotes differentiation with respect to $q^{2}$. The appearance of this maximum is inextricably connected with the presence of the unprotected logarithm originating from the ghost loop. In addition to confirming the known nonperturbative behavior
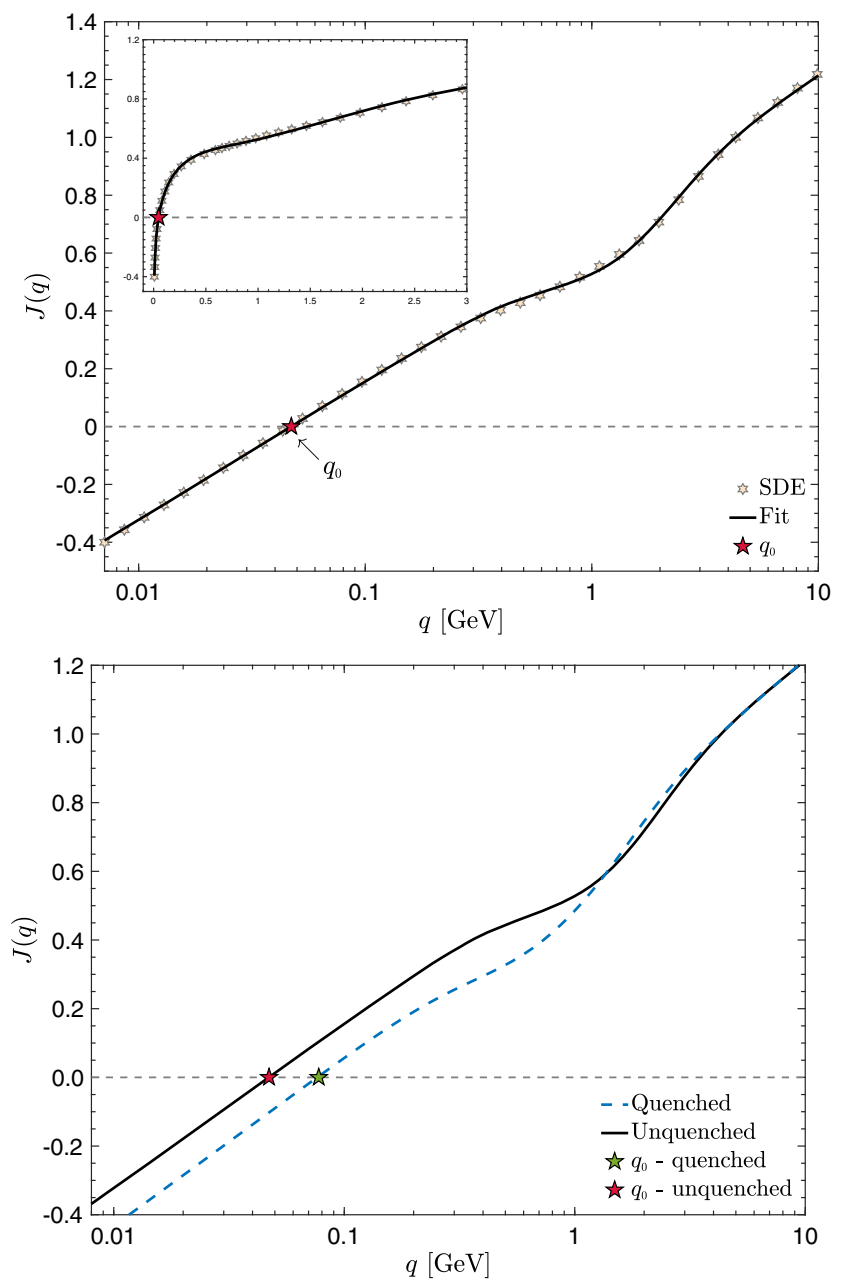

(black continuous), with the lattice data (solid circles in different colors for each $\beta$ ). Bottom right panel: The quenched (blue dashed) and unquenched (black continuous) $J(q)$; the stars indicate the momentum $q_{0}$ where $J\left(q_{0}\right)=0$

of the ghost propagator in Euclidean space (i.e., absence of a "ghost mass"), it has a direct implication on the general analytic structure of the gluon propagator $[49,94]$. In particular, from the standard Källén-Lehmann representation [95,96]

$\Delta\left(q^{2}\right)=\int_{0}^{\infty} d t \frac{\rho(t)}{q^{2}+t}$,

where $\rho(t)$ is the gluon spectral function, we have that

$\Delta^{\prime}\left(q^{2}\right)=-\int_{0}^{\infty} d t \frac{\rho(t)}{\left(q^{2}+t\right)^{2}}$.

Then, the maximum for $\Delta\left(q^{2}\right)$ at $q^{2}=q_{*}^{2}$ leads necessarily to positivity violation [13,97-99], because the condition

$\int_{0}^{\infty} d t \frac{\rho(t)}{\left(q_{*}^{2}+t\right)^{2}}=0$,

may be fulfilled only if $\rho(t)$ is not positive-definite. 
A reasonable estimate for the value of $q_{*}$ may be derived from Eq. (3.12); specifically one obtains the equation

$\left[\Delta^{-1}\left(q^{2}\right)\right]^{\prime}=a \ln \left(\frac{q^{2}}{\mu^{2}}\right)+\tilde{c}=0$,

where $\tilde{c}:=a+b+c$, whose solution is given by

$q_{*}=\mu \exp \left(-\frac{\tilde{c}}{2 a}\right)$,

yielding the numerical value $q_{*}=63 \mathrm{MeV}$.

The expression for the gluon propagator at the maximum is given by

$\Delta_{*}:=\Delta\left(q_{*}^{2}\right)=\left[d-a \mu^{2} \exp \left(-\frac{\tilde{c}}{a}\right)\right]^{-1}$,

its numerical value is given by $\Delta_{*}=5.28 \mathrm{GeV}^{-2}$.

(iv) Finally, we turn to another characteristic feature associated with the presence of the unprotected logarithm, namely the logarithmic divergence of $\Delta^{\prime}\left(q^{2}\right)$ at the origin. In particular, using Eq. (3.12), it is straightforward to establish that

$$
\begin{aligned}
& \Delta^{\prime}\left(q^{2}\right) \underset{q^{2} \rightarrow 0}{\simeq}-\frac{a}{d^{2}} \ln \left(\frac{q^{2}}{\mu^{2}}\right) \rightarrow+\infty, \\
& {\left[\Delta^{-1}\left(q^{2}\right)\right]^{\prime} \underset{q^{2} \rightarrow 0}{\simeq} a \ln \left(\frac{q^{2}}{\mu^{2}}\right) \rightarrow-\infty .}
\end{aligned}
$$

(v) While the functional form of $\Delta^{-1}\left(q^{2}\right)$ is motivated by sound theoretical considerations, the numerical values for the parameters $a, b, c$, and $d$, quoted below Eq. (3.13), have been obtained by fitting the entire range of the SDE solution. It would be therefore interesting to probe the stability of our asymptotic results by contrasting them directly with the low-momentum domain of the lattice data, and subsequently refitting the aforementioned parameters. To that end, we consider only the lattice ensemble with $\beta=1.63$, because it contains the largest number of points in the desired region. Our fitting procedure is limited to the data below a given momentum cutoff, $q_{\text {cut }}$; we have chosen two values for it, namely $q_{\text {cut }}=0.3 \mathrm{GeV}$ and $q_{\text {cut }}=0.4 \mathrm{GeV}$. The result of this analysis can be found in Fig. 3 and in Table 2.

The black continuous line corresponds to the SDE-based result, while the red dashed curve is its asymptotic limit. All asymptotic curves are obtained with Eq. (3.12) using the fitting parameters listed in the Table 2.

As we can see in Fig. 3, the asymptotic expression of Eq. (3.12) describes the lattice data particularly well. Essentially, the difference between the asymptotic limit of the SDE result (red dashed line) and the best fits for the IR lattice points (green band) appears for very low momenta, and is of the order of $3 \%$. The lattice data for $\Delta^{-1}\left(q^{2}\right)$ show a linear behavior, consistent with a $q^{2}$-increase, except for momenta below $180 \mathrm{MeV}$, where the effect of the logarithm in Eq. (3.12) becomes apparent. Note also the onset of a

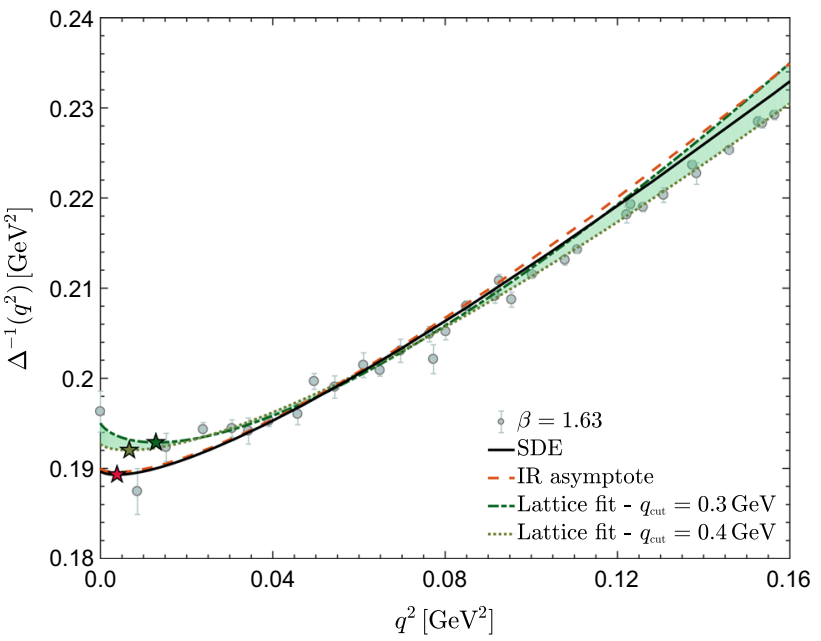

Fig. 3 The green band represents the asymptotic fits for the lattice data (gray solid circles) with $q_{\text {cut }}=0.3 \mathrm{GeV}$ (green dash-dotted line) and $q_{\text {cut }}=0.4 \mathrm{GeV}$ (green dashed curve), given by Eq. (3.12). The black continuous line corresponds to the SDE-based result, while the red dashed curve is its asymptotic limit. All asymptotic curves are given by Eq. (3.12), with the corresponding fitting parameters listed in the Table 2

steep derivative close to the origin, in qualitative agreement with point (iv). In addition, the refitted values of $a, b+c$, and $d$ are completely consistent with those obtained from the full-range fit of the SDE result.

\section{IR suppression of the three-gluon vertex}

In this section we present the SDE-based computation of $\bar{\Gamma}_{1}^{\text {sym }}\left(s^{2}\right)$ and $\bar{\Gamma}_{3}^{\text {asym }}\left(q^{2}\right)$. After an instructive study of the low-momentum limit, our results for the entire range of momenta are presented and compared with the new lattice data. In addition, the two effective couplings obtained from $\bar{\Gamma}_{1}^{\text {sym }}\left(s^{2}\right)$ and $\bar{\Gamma}_{3}^{\text {asym }}\left(q^{2}\right)$ are constructed, and the former is compared with the corresponding quantity obtained from the ghost-gluon vertex.

\subsection{The SDE-based derivation}

The detailed form of the function $J\left(q^{2}\right)$ captured by Eq. (3.9) constitutes a key ingredient for the approximate evaluation of the vertex form factors $\bar{\Gamma}_{1}^{\mathrm{sym}}\left(s^{2}\right)$ and $\bar{\Gamma}_{3}^{\text {asym }}\left(q^{2}\right)$ by means of the main equations Eq. (2.20) and Eq. (2.24), respectively. This becomes possible because the nonperturbative $\mathrm{BC}$ construction of [31] allows one to express the $X_{i}$ in terms of the kinetic term of the gluon propagator, the ghost dressing function, and the ghost-gluon form factors. Even though this procedure does not determine the terms $\left(s^{4} / 4\right) Y_{1}\left(s^{2}\right)-\left(s^{2} / 2\right) Y_{4}\left(s^{2}\right)$ contributing to $\bar{\Gamma}_{1}^{\text {sym }}\left(s^{2}\right)$, the overall agreement with the (admittedly error-burdened) lat- 
Table 2 Fitting parameters of Eq. (3.12) for different values of the cutoff $q_{\text {cut }}$ considered in the fitting process of the lattice data with $\beta=1.63$. In the last line, we show the fitting parameters of the SDE asymptotic limit, given by Eq. (3.12), which was obtained by expanding the result given in Eqs. (3.8) and (3.9). In the last column, we quote the momentum $q_{*}$, where the minimum of $\Delta^{-1}\left(q^{2}\right)$ [or maximum of $\Delta\left(q^{2}\right)$ ] occurs for each case

\begin{tabular}{llllr}
\hline IR asymptotic fits & $a$ & $b+c$ & $d\left(\mathrm{GeV}^{2}\right)$ & $q_{*}(\mathrm{MeV})$ \\
\hline Lattice $-q_{\text {cut }}=0.3(\mathrm{GeV})$ & 0.165 & 1.036 & 0.195 & 113 \\
Lattice $-q_{\text {cut }}=0.4[\mathrm{GeV}]$ & 0.107 & 0.746 & 0.193 & 82 \\
SDE expansion & 0.104 & 0.774 & 0.190 & 63 \\
\hline
\end{tabular}

tice results suggests that their omission does not alter drastically the qualitative features of the BC solution; see also the related discussions in Sect. 4.3.

Focusing precisely on $\bar{\Gamma}_{1}^{\mathrm{sym}}\left(s^{2}\right)$, the part that depends on the two longitudinal components is given by [31]

$$
\begin{aligned}
X_{1}\left(s^{2}\right)-\frac{s^{2}}{2} X_{3}\left(s^{2}\right)= & F\left(s^{2}\right)\left[J\left(s^{2}\right)\left(H_{1}\left(s^{2}\right)+\frac{s^{2}}{2} H_{3}\left(s^{2}\right)\right)\right. \\
& \left.+\frac{s^{2}}{2} \frac{d J\left(s^{2}\right)}{d s^{2}} H_{2}\left(s^{2}\right)\right]
\end{aligned}
$$

where

$$
\begin{aligned}
H_{1}\left(s^{2}\right)= & A_{1}\left(s^{2}\right)-\frac{s^{2}}{2} A_{3}\left(s^{2}\right), \\
H_{2}\left(s^{2}\right)= & A_{1}\left(s^{2}\right)+\frac{s^{2}}{2}\left[A_{3}\left(s^{2}\right)-A_{4}\left(s^{2}\right)\right], \\
H_{3}\left(s^{2}\right)= & A_{1}^{(1,0,0)}\left(s^{2}\right)+\frac{\sqrt{3}}{2 s^{2}} A_{1}^{(0,0,1)}\left(s^{2}\right) \\
& +\frac{s^{2}}{2}\left[A_{3}^{(1,0,0)}\left(s^{2}\right)-A_{4}^{(1,0,0)}\left(s^{2}\right)\right] \\
& +\frac{\sqrt{3}}{4}\left[A_{3}^{(0,0,1)}\left(s^{2}\right)-A_{4}^{(0,0,1)}\left(s^{2}\right)\right],
\end{aligned}
$$

with the partial derivatives defined as

$$
\begin{aligned}
& A_{i}^{(1,0,0)}\left(s^{2}\right)=\left.\frac{\partial A_{i}\left(q^{2}, r^{2}, \theta\right)}{\partial q^{2}}\right|_{q^{2}=r^{2}=s^{2}, \theta=2 \pi / 3}, \\
& A_{i}^{(0,1,0)}\left(s^{2}\right)=\left.\frac{\partial A_{i}\left(q^{2}, r^{2}, \theta\right)}{\partial r^{2}}\right|_{q^{2}=r^{2}=s^{2}, \theta=2 \pi / 3}, \\
& A_{i}^{(0,0,1)}\left(s^{2}\right)=\left.\frac{\partial A_{i}\left(q^{2}, r^{2}, \theta\right)}{\partial \theta}\right|_{q^{2}=r^{2}=s^{2}, \theta=2 \pi / 3} .
\end{aligned}
$$

Analogous relations, not reported here, hold for the asymmetric configuration.

Before turning to the full construction of $\bar{\Gamma}_{1}^{\text {sym }}\left(s^{2}\right)$, we focus on certain global aspects that it displays at low momenta, which may be obtained from the above expressions with a moderate amount of effort.

\subsection{The low-momentum limit}

In particular, Eq. (4.1) allows one to deduce the exact functional form of $\bar{\Gamma}_{1}^{\text {sym }}\left(s^{2}\right)$ in the limit $s^{2} \rightarrow 0$. Indeed, a preliminary one-loop dressed analysis reveals that, in that limit, the combination $\left(s^{4} / 4\right) Y_{1}\left(s^{2}\right)-\left(s^{2} / 2\right) Y_{4}\left(s^{2}\right)$ yields a constant term, to be denoted by $C_{t}$. Moreover, $s^{2} H_{3}\left(s^{2}\right), s^{2} A_{3}\left(s^{2}\right)$ and $s^{2} A_{4}\left(s^{2}\right)$ vanish, while $A_{1}(0)=1$, by virtue of the Taylor theorem [100]. Consequently, the leading contribution originates from the combination $F\left(s^{2}\right)\left[J\left(s^{2}\right)+\left(s^{2} / 2\right) J^{\prime}\left(s^{2}\right)\right]$.

Then, it is straightforward to establish from Eq. (3.9) that $\lim _{s^{2} \rightarrow 0} s^{2} J^{\prime}\left(s^{2}\right)=a$. Thus, the asymptotic form of $\bar{\Gamma}_{1}^{\text {sym }}\left(s^{2}\right)$ is given by

$$
\begin{gathered}
\bar{\Gamma}_{1}^{\operatorname{sym}}\left(s^{2}\right) \underset{s^{2} \rightarrow 0}{\simeq} F(0)\left[a \ln \left(\frac{s^{2}}{\mu^{2}}\right)+b+\frac{a}{2}\right] \\
=\tilde{a} \ln \left(\frac{s^{2}}{\mu^{2}}\right)+\tilde{b},
\end{gathered}
$$

where we have set $C_{t}=0$. Then, using the fact that the saturation value of the ghost dressing function is $F(0)=$ 2.92 when one renormalizes at $\mu=4.3 \mathrm{GeV}$, together with the values for $a$ and $b$ quoted below Eq. (3.13), one finds $\tilde{a}=0.303$ and $\tilde{b}=2.87$.

In the asymmetric case, a similar procedure may be employed to fully determine the behavior of $\bar{\Gamma}_{3}^{\text {asym }}\left(q^{2}\right)$ for small $q^{2}$, leading to

$$
\begin{gathered}
\bar{\Gamma}_{3}^{\operatorname{asym}}\left(q^{2}\right) \underset{q^{2} \rightarrow 0}{\simeq} F(0)\left[J\left(q^{2}\right)+\lim _{q^{2} \rightarrow 0} q^{2} J^{\prime}\left(q^{2}\right)\right] \\
=\tilde{a} \ln \left(\frac{q^{2}}{\mu^{2}}\right)+(b+a) F(0) .
\end{gathered}
$$

It is important to clarify at this point that, in a bona fide SDE analysis of the three-gluon vertex [21,24,26,28,101103], the asymptotic behavior found in Eq. (4.4) emerges from the ghost triangle diagram, shown in Fig. 4, which furnishes an unprotected logarithm. Of course, in the BC

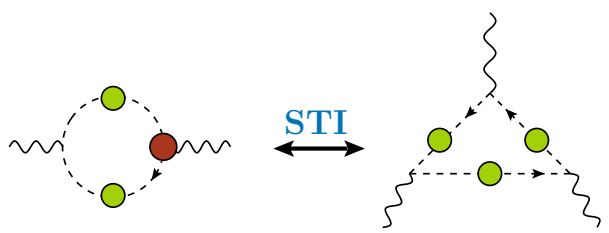

Fig. 4 The ghost loop diagram contributing to the kinetic term $J\left(q^{2}\right)$, and the ghost triangle diagram entering in the skeleton expansion of three-gluon vertex. Both diagrams are connected by the STI of Eq. (3.5), which imposes the equality of the corresponding unprotected logarithms 
construction followed in [31] and here, no vertex diagrams are considered; instead, the corresponding unprotected logarithm originates from the ghost loop diagram contributing to $J\left(q^{2}\right)$, shown in Fig. 4, which is related to the ghost triangle diagram by the STI of Eq. (3.5), as shown schematically in Fig. 4.

Note that the logarithms appearing in both Eq. (4.4) and Eq. (4.5) are multiplied by the same coefficient, namely $\tilde{a}$; this is a direct consequence of the fact that, in the Landau gauge, the ghost-gluon scattering kernel, $H_{\nu \mu}$, assumes its tree level value when the momentum of its ghost leg vanishes, in compliance with the well-known Taylor theorem. In particular, the $A_{i}$ enter into the $\mathrm{BC}$ solution with various permutations of $(q, r, p)$ in their arguments. Since in both cases considered all momenta eventually vanish, the substitution $A_{1} \rightarrow 1$ and $t^{2} A_{i} \rightarrow 0$ with $i=2,3,4,5$ is eventually triggered, where $t$ denotes any of these momenta. ${ }^{3}$ Specifically, one gets

$$
\begin{aligned}
& X_{1}\left(s^{2}\right) \underset{s^{2} \rightarrow 0}{\simeq} F\left(s^{2}\right) J\left(s^{2}\right), \\
& X_{3}\left(s^{2}\right) \underset{s^{2} \rightarrow 0}{\simeq}-F\left(s^{2}\right) J^{\prime}\left(s^{2}\right), \\
& X_{1}\left(q^{2}, q^{2}, \pi\right) \underset{q^{2} \rightarrow 0}{\simeq} F\left(q^{2}\right) J\left(q^{2}\right), \\
& X_{3}\left(q^{2}, q^{2}, \pi\right) \underset{q^{2} \rightarrow 0}{\simeq}-F(0) J^{\prime}\left(q^{2}\right),
\end{aligned}
$$

and the results of Eqs. (4.4) and (4.5) follow straightforwardly.

Note that, within a self-consistent renormalization scheme, the coefficient $\tilde{a}$ multiplying the IR divergent logarithm is common to both $\bar{\Gamma}_{3}^{\text {asym }}\left(q^{2}\right)$ and $\bar{\Gamma}_{3}^{\text {asym }}\left(q^{2}\right)$. However, the conditions $\bar{\Gamma}_{1}^{\text {sym }}\left(\mu^{2}\right)=\bar{\Gamma}_{3}^{\text {asym }}\left(\mu^{2}\right)=1$, enforced on the lattice data, cannot be simultaneously accommodated within a single scheme. Thus, the corresponding $\tilde{a}$ differ by a finite renormalization constant, which deviates very slightly from unity.

Let us finally point out that the qualitative analysis presented in this subsection remains valid even when $C_{t} \neq 0$, except for the location of the zero crossing, which will be shifted in a direction and by an amount that depend on the sign and size of this constant.

\subsection{Comparison with the lattice and further discussion}

Next, we proceed to the full determination of $\bar{\Gamma}_{1}^{\mathrm{sym}}\left(s^{2}\right)$ and $\bar{\Gamma}_{3}^{\text {asym }}\left(q^{2}\right)$ from the set of formulas given above [in particular Eqs. (2.20) and (2.24), together with Eqs. (4.1) (4.2)

\footnotetext{
3 The equality of the leading logarithms holds also perturbatively; however, in general, the $A_{i}$ cannot be set individually to their tree-level values, due to their higher rate of IR divergence. Nonperturbatively, the presence of a gluon mass scale attenuates these divergences [56], thus validating these substitutions.
}

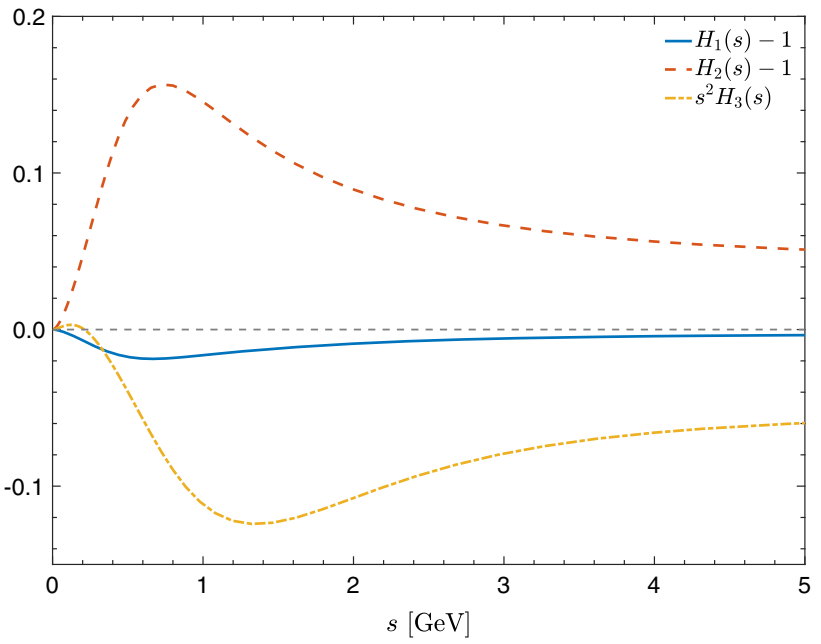

Fig. 5 The deviations of the combinations $H_{1}\left(s^{2}\right), H_{2}\left(s^{2}\right)$, and $s^{2} H_{3}\left(s^{2}\right)$, defined in the Eq. (4.2), from their tree-level counterparts

and (4.3)]. In order to accomplish this task, the functions $H_{1}\left(s^{2}\right), H_{2}\left(s^{2}\right)$, and $s^{2} H_{3}\left(s^{2}\right)$ must be computed from their defining equations, given in Eq. (4.2). This, in turn, requires the determination of the form factors $A_{1}\left(s^{2}\right), A_{3}\left(s^{2}\right)$, and $A_{4}\left(s^{2}\right)$, and the corresponding derivatives; since the impact of the unquenching effects on the ghost sector is expected to be rather small [36], for simplicity we use the quenched $A_{i}$ of [56]. The final $H_{1}\left(s^{2}\right), H_{2}\left(s^{2}\right)$, and $s^{2} H_{3}\left(s^{2}\right)$ are shown in Fig. 5, for the symmetric configuration; similar results have been obtained for the asymmetric case (not shown).

The comparison between the final SDE-based prediction for $\bar{\Gamma}_{1}^{\text {sym }}\left(s^{2}\right)$ and $\bar{\Gamma}_{3}^{\text {asym }}\left(q^{2}\right)$ and the corresponding unquenched lattice data is shown in Fig. 6; we observe a very good agreement for the entire range of momenta. It is rather evident that the particular shape of $J\left(q^{2}\right)$, shown in the top right panel of Fig. 2 and given by Eq. (3.9), is largely responsible for the most characteristic features of the vertex form factor at intermediate and low momenta, namely its overall suppression with respect to its tree-level value, and the inevitable (albeit hard to observe) reversal of sign (zero crossing) in the deep IR.

It is clear that, due to the well-known ambiguities related with the scale setting [104-107], direct comparisons between quenched and unquenched data may be quantitatively subtle. Notwithstanding this caveat, the inclusion of quarks seems to moderate the amount of suppression with respect to [19]. Specifically, the decrease observed between the renormalization point of $\mu=4.3 \mathrm{GeV}$ [where $\bar{\Gamma}_{1}^{\text {sym }}\left(\mu^{2}\right)=$ $\left.\bar{\Gamma}_{3}^{\operatorname{asym}}\left(\mu^{2}\right)=1\right]$ and a typical IR momentum, say $q_{I R}=300$ $\mathrm{MeV}$, is given by $\bar{\Gamma}_{1}^{\mathrm{sym}}\left(q_{I R}^{2}\right)=0.47$ and $\bar{\Gamma}_{1}^{\mathrm{asym}}\left(q_{I R}^{2}\right)=0.26$, to be compared with $\bar{\Gamma}_{1}^{\text {sym }}\left(q_{I R}^{2}\right)=0.33$ and $\bar{\Gamma}_{1}^{\text {asym }}\left(q_{I R}^{2}\right)=0.2$ for the quenched case; thus, the observed suppression is reduced by about $25 \%$. In addition, as expected from the 


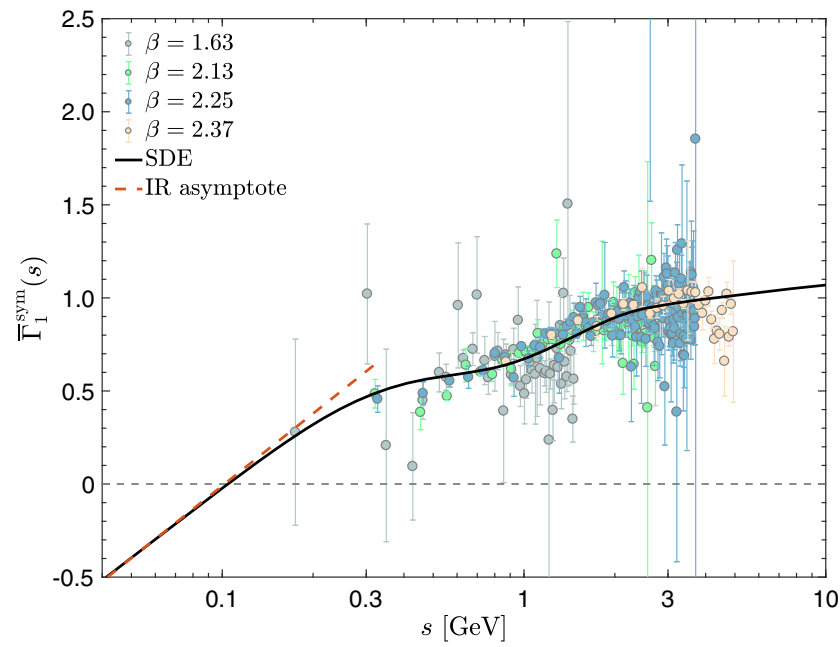

Fig. 6 Left panel: The form factor of the three-gluon vertex in the symmetric configuration, $\bar{\Gamma}_{1}^{\text {sym }}(s)$, obtained from lattice QCD (circles in different colors for each setup) and from the SDE-based approach

corresponding displacement of $q_{0}$ at the level of the $J\left(q^{2}\right)$ [see Sect. 3.3, point (i)], the zero crossing of both vertex configurations occurs at momenta lower compared to the quenched case, in qualitative agreement with the analysis of [28]. In particular, we find that the zero crossing moves from about $150 \mathrm{MeV}$ down to $105 \mathrm{MeV}$ (symmetric case) and from roughly $240 \mathrm{MeV}$ to about $170 \mathrm{MeV}$ (asymmetric case).

We next study in more detail the impact of the unprotected logarithm of $J\left(q^{2}\right)$ on the IR behavior of the vertex. In particular, $\bar{\Gamma}_{1}^{\text {sym }}\left(s^{2}\right)$ is computed by plugging into Eq. (4.1) (i) the full $J\left(q^{2}\right)$ given in Eq. (3.9) (black continuous curve), and (ii) a $J\left(q^{2}\right)$ without the term $(1 / 6) \ln \left(q^{2} / \mu^{2}\right)$ (red dashed curve). As we can see in the top left panel of Fig. 7, for momenta below $800 \mathrm{MeV}$ the unprotected logarithm starts to dominate the behavior of $\bar{\Gamma}_{1}^{\text {sym }}\left(s^{2}\right)$, forcing not only its suppression but also its IR divergence. In the remaining panels of Fig. 7 we show that the three sets of lattice data considered exhibit individually a clear preference for case (i); in fact, even in the least favorable case (top right panel), where the data are rather sparse and with sizable errors, the $\chi^{2} /$ d.o.f. is 1.8 times smaller than that of case (ii).

Finally, turning to the transverse part of $\Gamma_{\alpha \mu \nu}$, it is clear that the corresponding form factors ought to be determined from a detailed SDE study, which is still pending. In fact, the good coincidence found between the SDE-based prediction [with $Y_{1}\left(s^{2}\right)=Y_{4}\left(s^{2}\right)=0$ ] and the lattice must be interpreted with caution, especially in view of the sizable errors assigned to the data. Indeed, given the present precision, one may easily envisage how reasonably sized transverse contributions could be rather comfortably accommodated, pro-

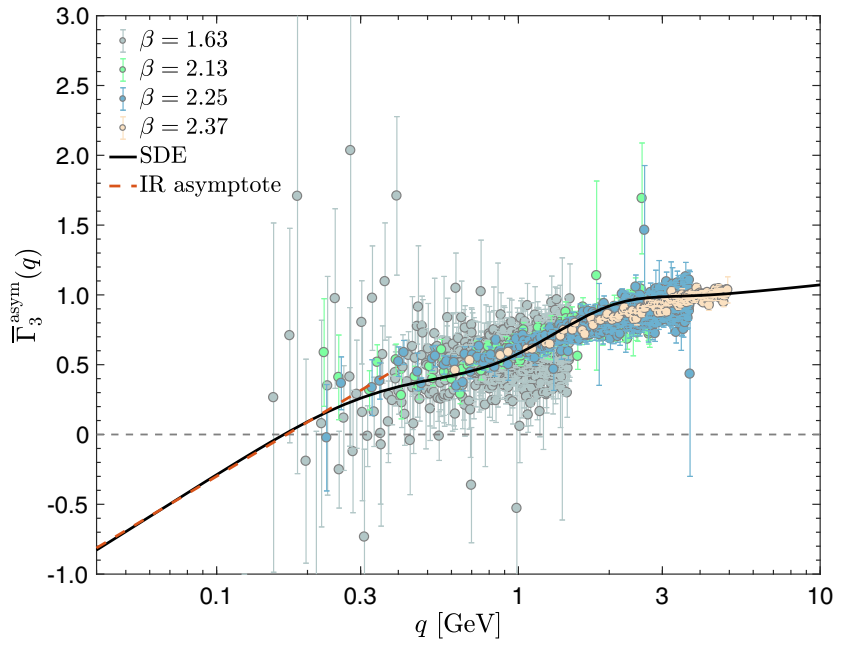

(black continuous curve). Right panel: The same for the asymmetric form factor $\bar{\Gamma}_{3}^{\text {asym }}(q)$. The IR asymptotes (red dashed lines) are given by Eqs. (4.4) and (4.5), respectively

vided they follow the general trend of the data. We hope to report progress in this direction in the near future.

\subsection{Effective couplings}

It is rather instructive to study how the suppression of the three-gluon vertex manifests itself at the level of a typical renormalization-group invariant quantity, which is traditionally used to quantify the effective strength of a given interaction.

To that end, we next consider the two effective couplings related to $\bar{\Gamma}_{1}^{\text {sym }}\left(s^{2}\right)$ and $\bar{\Gamma}_{3}^{\text {asym }}\left(q^{2}\right)$, to be denoted by $\widehat{g}^{\mathrm{sym}}\left(s^{2}\right)$ and $\widehat{g}^{\operatorname{asym}}\left(q^{2}\right)$, respectively. In particular, following standard definitions $[19,27,108]$, we have

$$
\begin{aligned}
& \widehat{g}^{\operatorname{sym}}\left(s^{2}\right)=g^{\operatorname{sym}}\left(\mu^{2}\right) s^{3} \bar{\Gamma}_{1}^{\operatorname{sym}}\left(s^{2}\right) \Delta^{3 / 2}\left(s^{2}\right), \\
& \widehat{g}^{\operatorname{asym}}\left(q^{2}\right)=g^{\operatorname{asym}}\left(\mu^{2}\right) q^{3} \bar{\Gamma}_{3}^{\text {asym }}\left(q^{2}\right) \Delta^{3 / 2}\left(q^{2}\right) .
\end{aligned}
$$

We emphasize that these two couplings may be recast in the form

$$
\begin{aligned}
& \widehat{g}^{\text {sym }}\left(s^{2}\right)=s^{3} \frac{T^{\text {sym }}\left(s^{2}\right)}{\Delta^{3 / 2}\left(s^{2}\right)}, \\
& \widehat{g}^{\text {asym }}\left(q^{2}\right)=q^{3} \frac{T^{\text {asym }}\left(q^{2}\right)}{\Delta(0) \Delta^{1 / 2}\left(q^{2}\right)},
\end{aligned}
$$

thus making contact with the corresponding definitions employed within the MOM schemes $[109,110]$. Turning to their computation, we use for the ingredients entering in the above definitions both lattice data as well as the corresponding SDE-derived quantities; the results obtained are displayed in Fig. 8. 

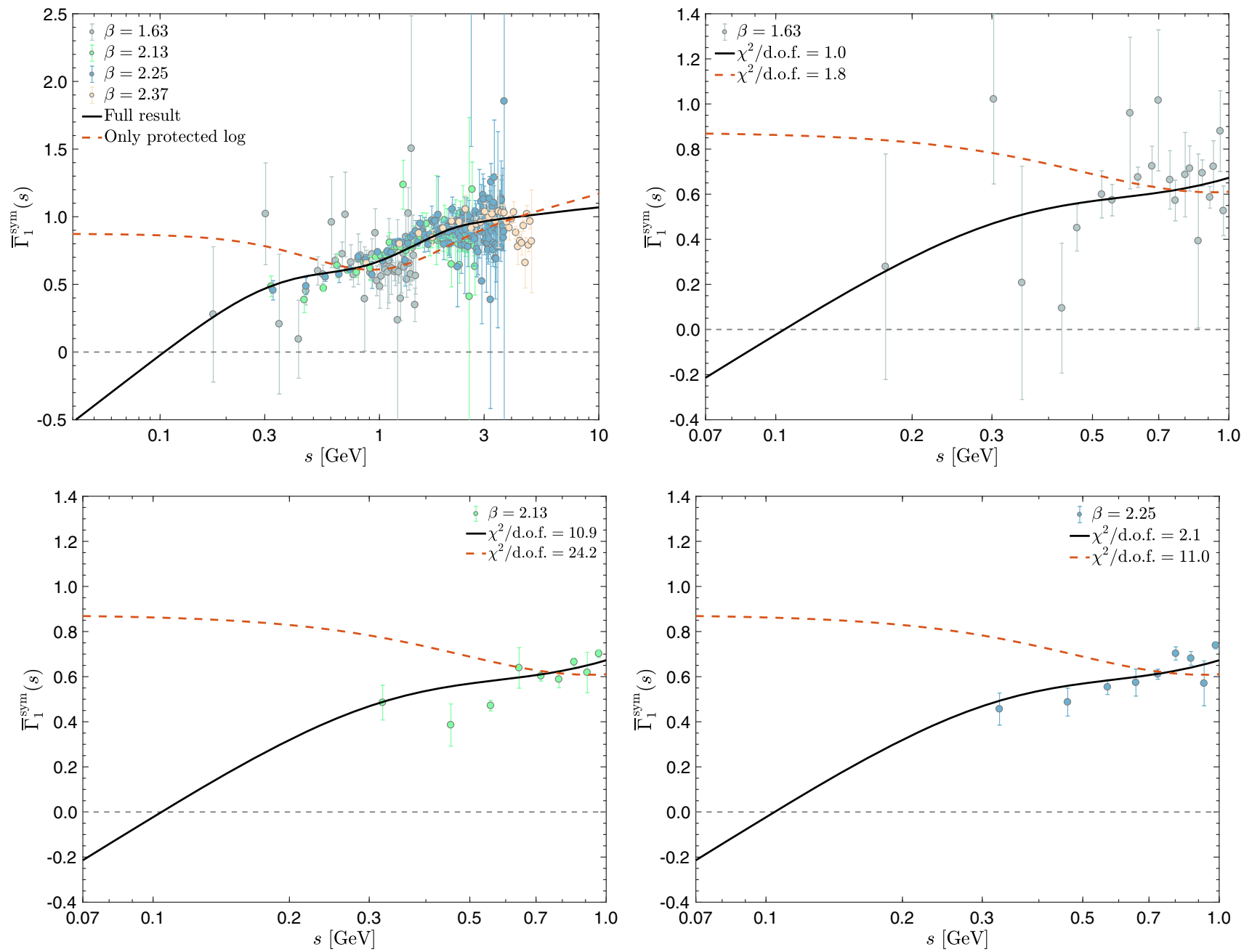

Fig. 7 Top left plot: the effect of the unprotected logarithm of $J\left(q^{2}\right)$ [see Eq. (3.9)] on the IR behavior of $\bar{\Gamma}_{1}^{\mathrm{sym}}(s)$. The black continuous line is $\bar{\Gamma}_{1}^{\text {sym }}(s)$ derived using the full expression for $J\left(q^{2}\right)$, given by Eq. (3.9). The red dashed curve represents the case where the massless logarithm, appearing in Eq. (3.9), is neglected. Top right plot: The

It is interesting to carry out a direct comparison of the effective coupling, $\widehat{g}^{\mathrm{sym}}\left(s^{2}\right)$, with the corresponding quantity, $\widehat{g}_{\mathrm{gh}}^{\mathrm{sym}}\left(s^{2}\right)$, associated with the ghost-gluon vertex in the symmetric configuration. Specifically, ${ }^{4}$

$\widehat{g}_{\text {gh }}^{\text {sym }}\left(s^{2}\right)=g^{\operatorname{sym}}\left(\mu^{2}\right) s \bar{B}_{1}^{\mathrm{sym}}\left(s^{2}\right) F\left(s^{2}\right) \Delta^{1 / 2}\left(s^{2}\right)$,

where $\bar{B}_{1}^{\mathrm{sym}}\left(s^{2}\right)$ denotes the form factor proportional to the tree-level component of the ghost-gluon vertex, renormalized at the same MOM point, $\mu=4.3 \mathrm{GeV}$. The functional form used for $\bar{B}_{1}^{\text {sym }}\left(s^{2}\right)$ has been obtained from the analysis of [56] and it is shown in the left panel of Fig. 9. The two

$\chi^{2} /$ d.o.f. when we compare the lattice data for $\beta=1.63$ with the results for $\bar{\Gamma}_{1}^{\text {sym }}(s)$ with (black continuous) and without (red dashed) the unprotected logarithm. Bottom left plot: The same as the previous panel but for the lattice data with $\beta=2.13$. Bottom right plot: Same analysis, using the lattice data with $\beta=2.25$

couplings are displayed in the left panel of Fig. 10; clearly, as the momentum $s$ decreases, $\widehat{g}^{\text {sym }}\left(s^{2}\right)$ becomes considerably smaller than $\widehat{g}_{\mathrm{gh}}^{\mathrm{sym}}\left(s^{2}\right)$.

In order to analyze in detail the origin of this relative suppression, it is advantageous to introduce the gluon dressing function, $\mathcal{Z}\left(q^{2}\right)$, defined as $\mathcal{Z}\left(q^{2}\right)=q^{2} \Delta\left(q^{2}\right)$, which is shown on the right panel of Fig. 9, together with the corresponding quantity for the ghost propagator, $F\left(q^{2}\right)$, introduced in Eq. (2.2). Then, the two effective couplings assume the form

${ }^{4}$ Using the formulas of [111], one finds that $g^{\text {sym }}\left(\mu^{2}\right) / g_{\text {gh }}^{\text {sym }}\left(\mu^{2}\right)=$ 1.03 at $\mu=4.3 \mathrm{GeV}$, which justifies the use of $g^{\text {sym }}\left(\mu^{2}\right)$ instead of $g_{\mathrm{gh}}^{\mathrm{sym}}\left(\mu^{2}\right)$ in the definition of Eq. (4.9).

$$
\begin{aligned}
& \widehat{g}^{\mathrm{sym}}\left(s^{2}\right)=g^{\mathrm{sym}}\left(\mu^{2}\right) \bar{\Gamma}_{1}^{\mathrm{sym}}\left(s^{2}\right) \mathcal{Z}^{3 / 2}\left(s^{2}\right), \\
& \widehat{g}_{\mathrm{gh}}^{\mathrm{sym}}\left(s^{2}\right)=g^{\operatorname{sym}}\left(\mu^{2}\right) \bar{B}_{1}^{\mathrm{sym}}\left(s^{2}\right) F\left(s^{2}\right) \mathcal{Z}^{1 / 2}\left(s^{2}\right) .
\end{aligned}
$$




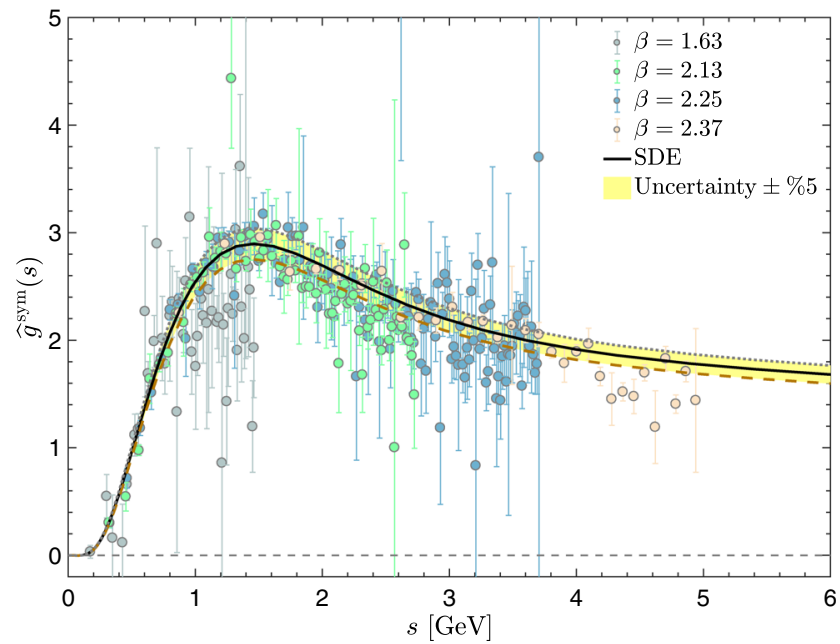

Fig. 8 Left panel: The symmetric effective coupling, $\widehat{g}^{\mathrm{sym}}(s)$, defined in Eq. (4.7), obtained using the lattice data (full circles) and the results of the SDE-based approach (black continuous line). The yellow band represents how the SDE result for $\widehat{g}^{\mathrm{sym}}(s)$ changes when the value

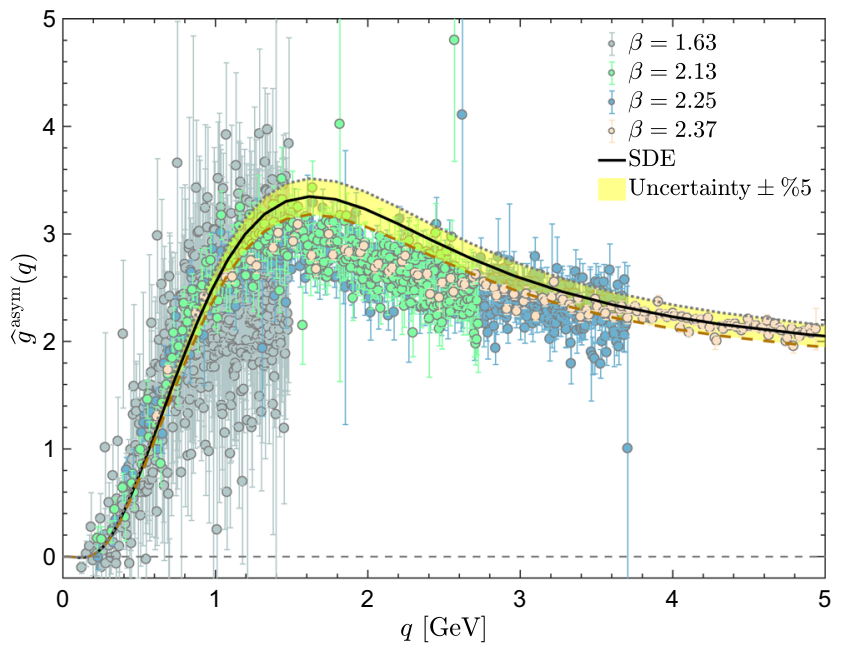

of $g$ at $\mu=4.3 \mathrm{GeV}$ has an uncertainty of $\pm 5 \%$, with central values $g^{\operatorname{sym}}\left(\mu^{2}\right)=1.86\left[\alpha^{\operatorname{sym}}\left(\mu^{2}\right)=0.27\right]$. Right panel: The same for the asymmetric effective coupling, $\widehat{g}^{\text {asym }}\left(q^{2}\right)$, with a central value $g^{\operatorname{asym}}\left(\mu^{2}\right)=2.16\left[\alpha^{\operatorname{asym}}\left(\mu^{2}\right)=0.37\right]$

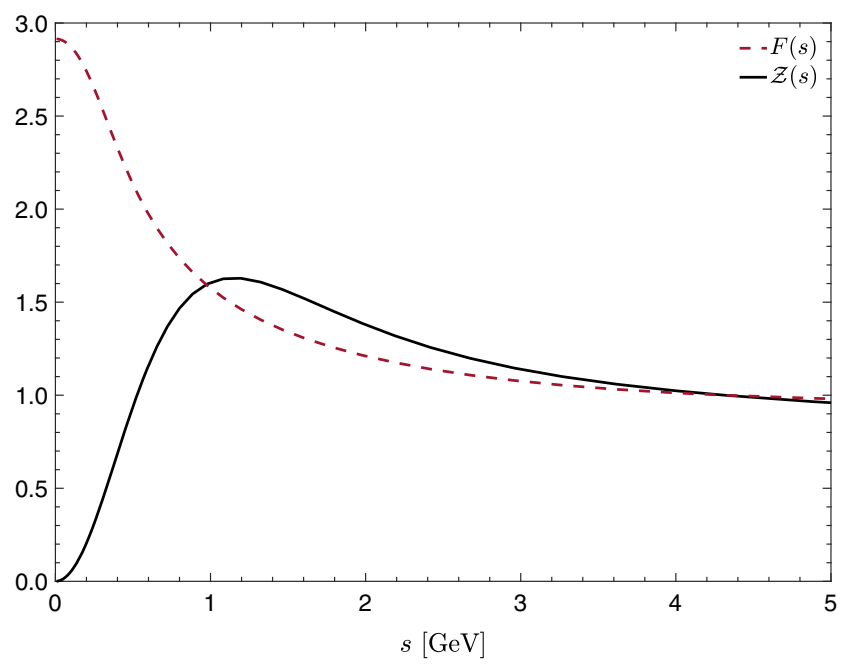

Fig. 9 Left panel: $\bar{\Gamma}_{1}^{\mathrm{sym}}(s)$ (black continuous line) compared with the form factor $\bar{B}_{1}^{\text {sym }}(s)$ of the ghost-gluon vertex (red dashed curve), in the symmetric configuration. Right panel: The gluon and ghost dressing functions, $\mathcal{Z}(s)$ and $F(s)$, respectively

We next consider the ratio of these two couplings,

$$
\begin{aligned}
\mathcal{R}_{g}\left(s^{2}\right) & =\widehat{g}^{\mathrm{sym}}\left(s^{2}\right) / \widehat{g}_{\mathrm{gh}}^{\mathrm{sym}}\left(s^{2}\right) \\
& =\underbrace{\left[\mathcal{Z}\left(s^{2}\right) / F\left(s^{2}\right)\right]}_{\mathcal{R}_{2}\left(s^{2}\right)} \underbrace{\left[\bar{\Gamma}_{1}^{\mathrm{sym}}\left(s^{2}\right) / \bar{B}_{1}^{\mathrm{sym}}\left(s^{2}\right)\right]}_{\mathcal{R}_{3}\left(s^{2}\right)},
\end{aligned}
$$

where the partial ratios $\mathcal{R}_{2}\left(s^{2}\right)$ and $\mathcal{R}_{3}\left(s^{2}\right)$ quantify the relative contribution from the two- and three-point sectors, respectively, at the various momentum scales involved. The three ratios, $\mathcal{R}_{g}\left(s^{2}\right), \mathcal{R}_{2}\left(s^{2}\right)$, and $\mathcal{R}_{3}\left(s^{2}\right)$ are shown on the right panel of Fig. 10.

Interestingly, $\mathcal{R}_{2}\left(s^{2}\right)$ and $\mathcal{R}_{3}\left(s^{2}\right)$ are smaller than 1 for $s<880 \mathrm{MeV}$ and $s<4.3 \mathrm{GeV}$, respectively. Therefore, in the region of momenta between $(0-880) \mathrm{MeV}$, the suppression of $\widehat{g}^{\mathrm{sym}}\left(s^{2}\right)$ emerges as a combined effect of both the two- and the three-point sectors, whereas, from $880 \mathrm{MeV}$ to $2.4 \mathrm{GeV}$ the suppression is exclusively due to the behavior of the three-gluon vertex.

\section{Discussion and conclusions}

In this article we have considered several nonperturbative aspects related to the gluon propagator, $\Delta\left(q^{2}\right)$, and the threegluon vertex, $\Gamma_{\alpha \mu \nu}$, in the context of Landau gauge QCD with $N_{f}=2+1$ dynamical quarks. Our approach combines 


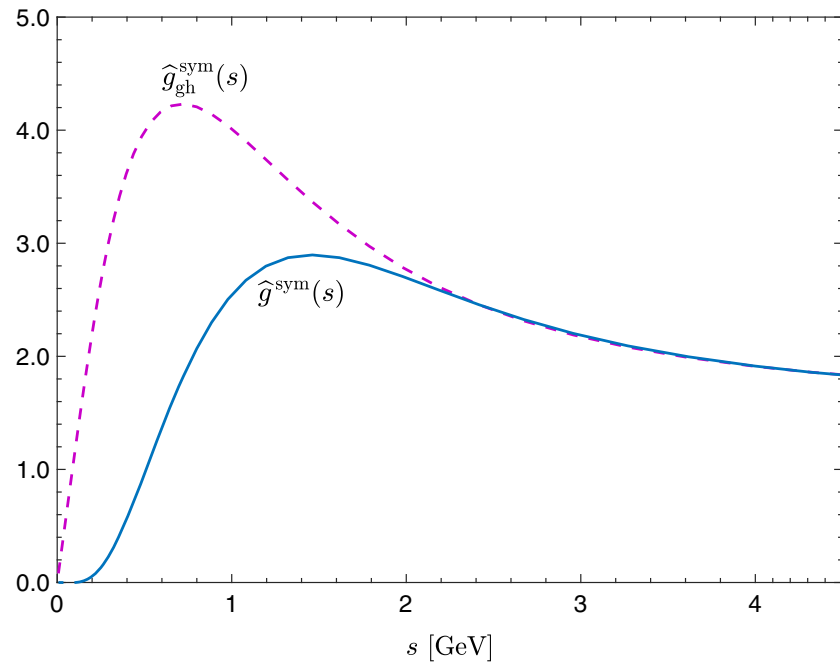

Fig. 10 Left panel: the comparison of the effective couplings, $\widehat{g}^{\text {sym }}(s)$ (blue solid line) and $\widehat{g}_{\mathrm{gh}}^{\mathrm{sym}}(s)$ (magenta dashed), defined from the threegluon vertex, Eq. (4.7), and from the ghost-gluon vertex, Eq. (4.9),

a SDE-based analysis, carried out within the PT-BFM framework, with new data gathered from lattice QCD simulations with $N_{f}=2+1$ domain wall fermions. In particular, from the SDE point of view, the gluon kinetic term $J\left(q^{2}\right)$ has been computed indirectly, by obtaining $m^{2}\left(q^{2}\right)$ from its own "gap equation" and then "subtracting" it from the new lattice data for $\Delta^{-1}\left(q^{2}\right)$. The $J\left(q^{2}\right)$ so determined is subsequently used for the "gauge technique" reconstruction (BC solution) of certain key form factors of $\Gamma_{\alpha \mu \nu}$, evaluated at two special kinematic configurations ("symmetric" and "asymmetric"). The two main quantities emerging from this construction, denoted by $\bar{\Gamma}_{1}^{\text {sym }}\left(s^{2}\right)$ and $\bar{\Gamma}_{3}^{\text {asym }}\left(q^{2}\right)$, are then compared with recently acquired lattice data, displaying very good coincidence. We emphasize that, while the determination of $J\left(q^{2}\right)$ hinges on the use of the lattice data for the gluon propagator, the subsequent results derived by means of this $J\left(q^{2}\right)$ constitute genuine theoretical predictions.

There are certain key theoretical notions underlying this work which are worth highlighting.

(i) The recent nonlinear SDE analysis of [61] generalizes from pure Yang-Mills to the case of real-world QCD with dynamical quarks, giving rise to a $m^{2}\left(q^{2}\right)$ that displays all qualitative features known from the quenched case.

(ii) The low-momentum behavior of $J\left(q^{2}\right)$ is clearly dominated by the unprotected logarithm originating from the ghost loop. In a pure Yang-Mills context, the diverging contribution of this logarithm overcomes the opposing action of its protected counterparts, leading to the IR suppression of $J\left(q^{2}\right)$ and its zero crossing. The inclusion of quark loops, which are regulated by the quark masses, gives rise to additional IR finite contributions, whose net effect is to attenuate the aforementioned outstanding features.

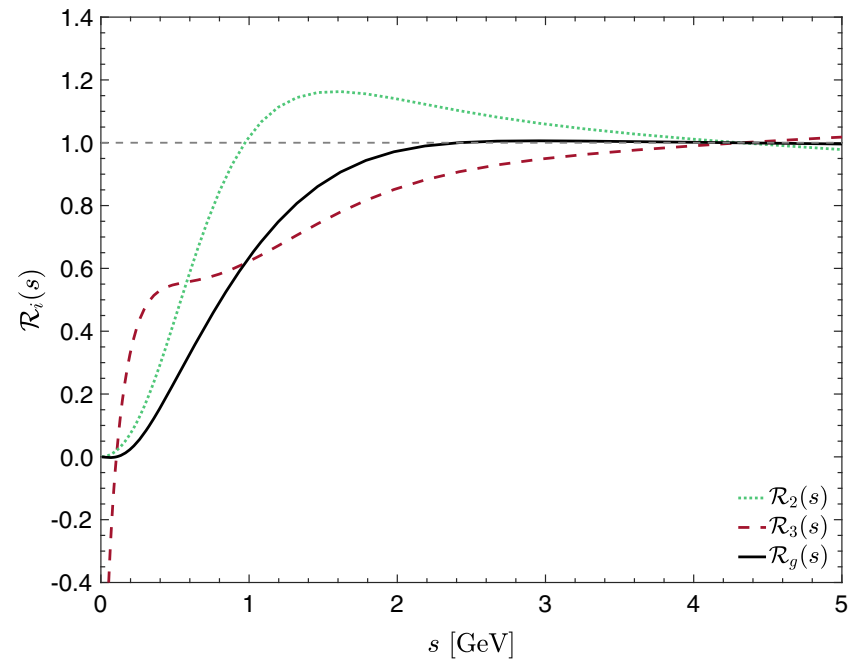

respectively. Right panel: the ratios $\mathcal{R}_{g}(s)$ (black continuous), $\mathcal{R}_{2}(s)$ (green dotted), and $\mathcal{R}_{3}(s)$ (red dashed) introduced in the Eq. (4.11)

(iii) By virtue of the fundamental STI of Eq. (3.5), the longitudinal form factors of $\Gamma_{\alpha \mu \nu}$ display the same qualitative characteristics as the $J\left(q^{2}\right)$; in that sense, the influence of the ghost sector, and in particular of the ghost-gluon kernel, is rather limited, and does not alter the main dynamical properties that $\bar{\Gamma}_{1}^{\text {sym }}\left(s^{2}\right)$ and $\bar{\Gamma}_{3}^{\text {asym }}\left(q^{2}\right)$ inherit from the $J\left(q^{2}\right)$.

(iv) In our opinion, the present analysis provides additional support for the picture of the IR sector of (Landau gauge) QCD that has emerged in recent years from a plethora of complementary considerations $[15,16,39,44,47]$. Specifically, the quarks acquire dynamically generated constituent masses, the ghosts remain strictly massless, while the gluons display the phenomenon of "gapping" $[29,80]$, according to which, the generation of a fundamental mass gap in the gauge sector enforces the property $\Delta(0)<\infty$. Within this latter context, one distinguishes "scaling solutions" $[13,14,39]$, for which $\Delta(0)=0$, from "decoupling solutions", with $\Delta(0)>0$; the latter type has been the focal point of the present work, with the mass function $m^{2}\left(q^{2}\right)$ implementing explicitly the IR saturation of $\Delta\left(q^{2}\right)$ at $m^{-2}(0)$. Evidently, the intricate structure and exceptional features displayed by $\Delta\left(q^{2}\right)$, together with the nontrivial dynamics needed for generating the aforementioned mass gap (such as the Schwinger mechanism), clearly differentiate the nonperturbative gluon from a naive "massive" gauge boson. The three-gluon vertex appears to be the host of an elaborate synergy between the mechanisms responsible for this exceptional mass patterns, thus providing an outstanding testing ground both for physics ideas as well as computational methods.

Acknowledgements We are very grateful to $\mathrm{Ph}$. Boucaud for his crucial help in obtaining the numerical data, during the early stages 
of this project, and to the RBC/UKQCD collaboration, especially P. Boyle, N. Christ, Z. Dong, C. Jung, N. Garron, B. Mawhinney and $\mathrm{O}$. Witzel, for access to the lattice configurations employed herein. Our lattice calculations benefited from the following resources: CINES, GENCI, IDRIS (Project ID 52271); and the IN2P3 Computing Facility. This work is supported by the Spanish Ministry of Economy and Competitiveness (MINECO) under Grants FPA2017-84543P (J.P.) and FPA2017-86380-P (J.R.-Q. and F.S.). J.P. also acknowledges the Generalitat Valenciana for the grant Prometeo/2019/087. The work of A.C.A. and M.N.F. is supported by the Brazilian National Council for Scientific and Technological Development (CNPq) under the Grants 305815/2015, 142226/2016-5, and 464898/2014-5 (INCTFNA). A.C.A. also acknowledges the financial support from São Paulo Research Foundation (FAPESP) through the project 2017/05685-2.

Data Availability Statement This manuscript has no associated data or the data will not be deposited. [Authors' comment: All data generated or analysed during this study are included in this published article.]

Open Access This article is licensed under a Creative Commons Attribution 4.0 International License, which permits use, sharing, adaptation, distribution and reproduction in any medium or format, as long as you give appropriate credit to the original author(s) and the source, provide a link to the Creative Commons licence, and indicate if changes were made. The images or other third party material in this article are included in the article's Creative Commons licence, unless indicated otherwise in a credit line to the material. If material is not included in the article's Creative Commons licence and your intended use is not permitted by statutory regulation or exceeds the permitted use, you will need to obtain permission directly from the copyright holder. To view a copy of this licence, visit http://creativecomm ons.org/licenses/by/4.0/.

Funded by SCOAP ${ }^{3}$.

\section{References}

1. W.J. Marciano, H. Pagels, Phys. Rep. 36, 137 (1978)

2. J.S. Ball, T.-W. Chiu, Phys. Rev. D 22, 2550 (1980)

3. A.I. Davydychev, P. Osland, O.V. Tarasov, Phys. Rev. D 54, 4087 (1996)

4. J.A. Gracey, Phys. Rev. D 84, 085011 (2011). https://doi.org/10. 1103/PhysRevD.84.085011

5. J.A. Gracey, H. Kißler, D. Kreimer, Phys. Rev. D 100(8), 085001 (2019)

6. J.M. Cornwall, Phys. Rev. D 26, 1453 (1982)

7. C.W. Bernard, Phys. Lett. B 108, 431 (1982)

8. C.W. Bernard, Nucl. Phys. B 219, 341 (1983)

9. J.F. Donoghue, Phys. Rev. D 29, 2559 (1984)

10. K.G. Wilson, T.S. Walhout, A. Harindranath, W.-M. Zhang, R.J. Perry, S.D. Glazek, Phys. Rev. D 49, 6720 (1994). https://doi.org/ 10.1103/PhysRevD.49.6720

11. O. Philipsen, Nucl. Phys. B 628, 167 (2002). https://doi.org/10. 1016/S0550-3213(02)00089-5

12. A.C. Aguilar, A.A. Natale, P.S. Rodrigues da Silva, Phys. Rev. Lett. 90, 152001 (2003)

13. R. Alkofer, L. von Smekal, Phys. Rep. 353, 281 (2001)

14. C.S. Fischer, J. Phys. G32, R253 (2006)

15. A.C. Aguilar, D. Binosi, J. Papavassiliou, Phys. Rev. D 78, 025010 (2008). https://doi.org/10.1103/PhysRevD.78.025010

16. P. Boucaud, J. Leroy, L.Y.A.J. Micheli, O. Pène, J. RodríguezQuintero, JHEP 06, 099 (2008). https://doi.org/10.1088/ 1126-6708/2008/06/099

17. A. Cucchieri, A. Maas, T. Mendes, Phys. Rev. D 74, 014503 (2006). https://doi.org/10.1103/PhysRevD.74.014503
18. A. Cucchieri, A. Maas, T. Mendes, Phys. Rev. D 77, 094510 (2008). https://doi.org/10.1103/PhysRevD.77.094510

19. A. Athenodorou, D. Binosi, P. Boucaud, F. De Soto, J. Papavassiliou, J. Rodriguez-Quintero, S. Zafeiropoulos, Phys. Lett. B 761, 444 (2016). https://doi.org/10.1016/j.physletb.2016.08.065

20. A.G. Duarte, O. Oliveira, P.J. Silva, Phys. Rev. D 94, 074502 (2016). https://doi.org/10.1103/PhysRevD.94.074502

21. M.Q. Huber, A. Maas, L. von Smekal, JHEP 11, 035 (2012). https://doi.org/10.1007/JHEP11(2012)035

22. M. Pelaez, M. Tissier, N. Wschebor, Phys. Rev. D 88, 125003 (2013). https://doi.org/10.1103/PhysRevD.88.125003

23. A.C. Aguilar, D. Binosi, D. Ibañez, J. Papavassiliou, Phys. Rev. D 89, 085008 (2014). https://doi.org/10.1103/PhysRevD.89. 085008

24. A. Blum, M.Q. Huber, M. Mitter, L. von Smekal, Phys. Rev. D 89, 061703 (2014). https://doi.org/10.1103/PhysRevD.89.061703

25. A.L. Blum, R. Alkofer, M.Q. Huber, A. Windisch, Acta Phys. Pol. Supp. 8, 321 (2015)

26. G. Eichmann, R. Williams, R. Alkofer, M. Vujinovic, Phys. Rev. D 89, 105014 (2014). https://doi.org/10.1103/PhysRevD.89. 105014

27. M. Mitter, J.M. Pawlowski, N. Strodthoff, Phys. Rev. D 91, 054035 (2015). https://doi.org/10.1103/PhysRevD.91.054035

28. R. Williams, C.S. Fischer, W. Heupel, Phys. Rev. D 93, 034026 (2016). https://doi.org/10.1103/PhysRevD.93.034026

29. A.K. Cyrol, L. Fister, M. Mitter, J.M. Pawlowski, N. Strodthoff, Phys. Rev. D 94, 054005 (2016). https://doi.org/10.1103/ PhysRevD.94.054005

30. L. Corell, A .K. Cyrol, M. Mitter, J .M. Pawlowski, N. Strodthoff, SciPost Phys. 5, 066 (2018). https://doi.org/10.21468/ SciPostPhys.5.6.066

31. A.C. Aguilar, M.N. Ferreira, C.T. Figueiredo, J. Papavassiliou, Phys. Rev. D 99, 094010 (2019a). https://doi.org/10.1103/ PhysRevD.99.094010

32. A. Cucchieri, T. Mendes, PoS LAT2007, 297 (2007)

33. I .L. Bogolubsky, E .M. Ilgenfritz, M. Muller-Preussker, A. Sternbeck, PoS LATTICE2007, 290 (2007)

34. I. Bogolubsky, E. Ilgenfritz, M. Muller-Preussker, A. Sternbeck, Phys. Lett. B 676, 69 (2009). https://doi.org/10.1016/j.physletb. 2009.04.076

35. O. Oliveira, P. Silva, PoS LAT2009, 226 (2009)

36. A. Ayala, A. Bashir, D. Binosi, M. Cristoforetti, J. RodriguezQuintero, Phys. Rev. D 86, 074512 (2012). https://doi.org/10. 1103/PhysRevD.86.074512

37. A.C. Aguilar, A.A. Natale, JHEP 08, 057 (2004)

38. A.C. Aguilar, J. Papavassiliou, JHEP 12, 012 (2006)

39. C.S. Fischer, A. Maas, J.M. Pawlowski, Ann. Phys. 324, 2408 (2009). https://doi.org/10.1016/j.aop.2009.07.009

40. D. Dudal, J.A. Gracey, S.P. Sorella, N. Vandersickel, H. Verschelde, Phys. Rev. D 78, 065047 (2008). https://doi.org/10.1103/ PhysRevD.78.065047

41. J. Rodriguez-Quintero, JHEP 01, 105 (2011). https://doi.org/10. 1007/JHEP01(2011)105

42. M. Tissier, N. Wschebor, Phys. Rev. D 82, 101701 (2010). https:// doi.org/10.1103/PhysRevD.82.101701

43. M. Pennington, D. Wilson, Phys. Rev. D 84, 119901 (2011). https://doi.org/10.1103/PhysRevD.84.094028, https:// doi.org/10.1103/PhysRevD.84.119901

44. I.C. Cloet, C.D. Roberts, Prog. Part. Nucl. Phys. 77, 1 (2014). https://doi.org/10.1016/j.ppnp.2014.02.001

45. L. Fister, J.M. Pawlowski, Phys. Rev. D 88, 045010 (2013). https:// doi.org/10.1103/PhysRevD.88.045010

46. A.K. Cyrol, M.Q. Huber, L. von Smekal, Eur. Phys. J. C 75, 102 (2015). https://doi.org/10.1140/epjc/s10052-015-3312-1

47. D. Binosi, L. Chang, J. Papavassiliou, C.D. Roberts, Phys. Lett. B 742, 183 (2015). https://doi.org/10.1016/j.physletb.2015.01.031 
48. A.C. Aguilar, D. Binosi, J. Papavassiliou, Front. Phys. (Beijing) 11, 111203 (2016)

49. A .K. Cyrol, J .M. Pawlowski, A. Rothkopf, N. Wink, SciPost Phys. 5, 065 (2018). https://doi.org/10.21468/SciPostPhys.5.6. 065

50. J.M. Cornwall, J. Papavassiliou, Phys. Rev. D 40, 3474 (1989)

51. A. Pilaftsis, Nucl. Phys. B 487, 467 (1997)

52. D. Binosi, J. Papavassiliou, Phys. Rep. 479, 1 (2009). https://doi. org/10.1016/j.physrep.2009.05.001

53. L.F. Abbott, Nucl. Phys. B 185, 189 (1981)

54. D. Binosi, J. Papavassiliou, Phys. Rev. D 77, 061702 (2008). https://doi.org/10.1103/PhysRevD.77.061702

55. D. Binosi, D. Ibañez, J. Papavassiliou, Phys. Rev. D 86, 085033 (2012). https://doi.org/10.1103/PhysRevD.86.085033

56. A.C. Aguilar, M.N. Ferreira, C.T. Figueiredo, J. Papavassiliou, Phys. Rev. D 99, 034026 (2019b). https://doi.org/10.1103/ PhysRevD.99.034026

57. P. Boucaud, F. De Soto, K. Raya, J. Rodríguez-Quintero, S. Zafeiropoulos, Phys. Rev. D 98, 114515 (2018). https://doi.org/ 10.1103/PhysRevD.98.114515

58. T. Blum et al., (RBC, UKQCD), Phys. Rev. D 93, 074505 (2016). https://doi.org/10.1103/PhysRevD.93.074505

59. P.A. Boyle et al., Phys. Rev. D 93, 054502 (2016). https://doi.org/ 10.1103/PhysRevD.93.054502

60. P.A. Boyle, L. Del Debbio, A. Jüttner, A. Khamseh, F. Sanfilippo, J.T. Tsang, JHEP 12, 008 (2017). https://doi.org/10.1007/ JHEP12(2017)008

61. A.C. Aguilar, M.N. Ferreira, C.T. Figueiredo, J. Papavassiliou, Phys. Rev. D 100, 094039 (2019c)

62. Y. Iwasaki, Nucl. Phys. B 258, 141 (1985). https://doi.org/10. 1016/0550-3213(85)90606-6

63. D.B. Kaplan, Phys. Lett. B 288, 342 (1992). https://doi.org/10. 1016/0370-2693(92)91112-M

64. Y. Shamir, Nucl. Phys. B 406, 90 (1993). https://doi.org/10.1016/ 0550-3213(93)90162-I

65. P.M. Vranas, Nucl. Phys. B Proc. Suppl. 94, 177 (2001)

66. D.B. Kaplan. arXiv:0912.2560 [hep-lat]

67. R.C. Brower, H. Neff, K. Orginos, Nucl. Phys. Proc. Suppl. 140, 686 (2005)

68. Z.-F. Cui, J.-L. Zhang, D. Binosi, F. de Soto, C. Mezrag, J. Papavassiliou, C.D. Roberts, J. Rodríguez-Quintero, J. Segovia, S. Zafeiropoulos, arXiv:1912.08232 [hep-ph]

69. S. Zafeiropoulos, P. Boucaud, F. De Soto, J. Rodríguez-Quintero, J. Segovia, Phys. Rev. Lett. 122, 162002 (2019). https://doi.org/ 10.1103/PhysRevLett.122.162002

70. P. Boucaud, F. De Soto, J. Rodríguez-Quintero, S. Zafeiropoulos, Phys. Rev. D 95, 114503 (2017). https://doi.org/10.1103/ PhysRevD.95.114503

71. D. Becirevic, P. Boucaud, J. Leroy, J. Micheli, O. Pene, J. Rodriguez-Quintero, C. Roiesnel, Phys. Rev. D 60, 094509 (1999). https://doi.org/10.1103/PhysRevD.60.094509

72. D. Becirevic, P. Boucaud, J. Leroy, J. Micheli, O. Pene, J. Rodriguez-Quintero, C. Roiesnel, Phys. Rev. D 61, 114508 (2000). https://doi.org/10.1103/PhysRevD.61.114508

73. F. de Soto, C. Roiesnel, JHEP 09, 007 (2007). https://doi.org/10. 1088/1126-6708/2007/09/007

74. A.C. Aguilar, D. Binosi, J. Papavassiliou, Phys. Rev. D 95, 034017 (2017). https://doi.org/10.1103/PhysRevD.95.034017

75. A. Cucchieri, T. Mendes, Phys. Rev. Lett. 100, 241601 (2008). https://doi.org/10.1103/PhysRevLett.100.241601

76. A. Cucchieri, T. Mendes, Phys. Rev. D 81, 016005 (2010). https:// doi.org/10.1103/PhysRevD.81.016005

77. P. Boucaud et al., JHEP 06, 001 (2006)

78. P.O. Bowman et al., Phys. Rev. D 76, 094505 (2007)
79. P. Bicudo, D. Binosi, N. Cardoso, O. Oliveira, P.J. Silva, Phys. Rev. D 92, 114514 (2015). https://doi.org/10.1103/PhysRevD.92. 114514

80. J. Braun, H. Gies, J.M. Pawlowski, Phys. Lett. B 684, 262 (2010). https://doi.org/10.1016/j.physletb.2010.01.009

81. D. Epple, H. Reinhardt, W. Schleifenbaum, A. Szczepaniak, Phys. Rev. D 77, 085007 (2008). https://doi.org/10.1103/PhysRevD.77. 085007

82. J. Serreau, M. Tissier, Phys. Lett. B 712, 97 (2012). https://doi. org/10.1016/j.physletb.2012.04.041

83. J.S. Schwinger, Phys. Rev. 125, 397 (1962a)

84. J.S. Schwinger, Phys. Rev. 128, 2425 (1962b)

85. A.C. Aguilar, D. Ibanez, V. Mathieu, J. Papavassiliou, Phys. Rev. D 85, 014018 (2012a). https://doi.org/10.1103/PhysRevD. 85.014018

86. D. Ibañez, J. Papavassiliou, Phys. Rev. D 87, 034008 (2013). https://doi.org/10.1103/PhysRevD.87.034008

87. D. Binosi, J. Papavassiliou, Phys. Rev. D 97, 054029 (2018) https://doi.org/10.1103/PhysRevD.97.054029

88. A.C. Aguilar, D. Binosi, C.T. Figueiredo, J. Papavassiliou, Eur. Phys. J. C 78, 181 (2018). https://doi.org/10.1140/epjc/ s10052-018-5679-2

89. J. Braun, L. Fister, J.M. Pawlowski, F. Rennecke, Phys. Rev. D 94, 034016 (2016). https://doi.org/10.1103/PhysRevD.94.034016

90. C. Fischer, P. Watson, W. Cassing, Phys. Rev. D 72, 094025 (2005). https://doi.org/10.1103/PhysRevD.72.094025

91. A.K. Cyrol, M. Mitter, J.M. Pawlowski, N. Strodthoff, Phys. Rev. D 97, 054006 (2018b). https://doi.org/10.1103/PhysRevD. 97.054006

92. A.C. Aguilar, D. Binosi, J. Papavassiliou, Phys. Rev. D 86, 014032 (2012b)

93. A.C. Aguilar, D. Binosi, J. Papavassiliou, Phys. Rev. D 88, 074010 (2013a). https://doi.org/10.1103/PhysRevD.88.074010

94. W. Kern, M.Q. Huber, R. Alkofer, Phys. Rev. D 100, 094037 (2019). https://doi.org/10.1103/PhysRevD.100.094037

95. G. Källén, Helv. Phys. Acta 25, 417 (1952). https://doi.org/10. 1007/978-3-319-00627-7_90

96. H. Lehmann, Nuovo Cim. 11, 342 (1954). https://doi.org/10. 1007/BF02783624

97. K. Osterwalder, R. Schrader, Commun. Math. Phys. 31, 83 (1973). https://doi.org/10.1007/BF01645738

98. K. Osterwalder, R. Schrader, Commun. Math. Phys. 42, 281 (1975). https://doi.org/10.1007/BF01608978

99. J.M. Cornwall, Mod. Phys. Lett. A 28, 1330035 (2013). https:// doi.org/10.1142/S0217732313300358

100. J.C. Taylor, Nucl. Phys. B 33, 436 (1971)

101. W. Schleifenbaum, A. Maas, J. Wambach, R. Alkofer, Phys. Rev. D 72, 014017 (2005)

102. M.Q. Huber, L. von Smekal, JHEP 04, 149 (2013). https://doi. org/10.1007/JHEP04(2013)149

103. A.C. Aguilar, D. Ibañez, J. Papavassiliou, Phys. Rev. D 87, 114020 (2013b). https://doi.org/10.1103/PhysRevD.87.114020

104. R. Sommer, Nucl. Phys. B 411, 839 (1994). https://doi.org/10. 1016/0550-3213(94)90473-1

105. S. Capitani, M. Lüscher, R. Sommer, H. Wittig, Nucl. Phys. B544, 669 (1999), [Erratum: Nucl. Phys. B582, 762 (2000)]. https://doi.org/10.1016/S0550-3213(00)00163-2, https://doi.org/10.1016/S0550-3213(98)00857-8

106. D. Becirevic, P. Boucaud, L. Giusti, J.P. Leroy, V. Lubicz, G. Martinelli, F. Mescia, F. Rapuano. arXiv:hep-lat/9809129

107. P. Boucaud et al., JHEP 04, 006 (2000). https://doi.org/10.1088/ 1126-6708/2000/04/006

108. W.-J. Fu, J.M. Pawlowski, F. Rennecke. arXiv:1909.02991 [hep$\mathrm{ph}]$ 
109. B. Alles, D. Henty, H. Panagopoulos, C. Parrinello, C. Pittori, D.G. Richards, Nucl. Phys. B 502, 325 (1997). https://doi.org/10. 1016/S0550-3213(97)00483-5

110. P. Boucaud, J.P. Leroy, J. Micheli, O. Pene, C. Roiesnel, JHEP 10, 017 (1998). https://doi.org/10.1088/1126-6708/1998/10/017
111. K. Chetyrkin, T. Seidensticker, Phys. Lett. B 495, 74 (2000). https://doi.org/10.1016/S0370-2693(00)01217-X 